\title{
Space-Time Rate Splitting for the MISO BC with Magnitude CSIT
}

\author{
Carlos Mosquera*, Nele Noels ${ }^{\dagger}$, Tomás Ramírez*, Màrius Caus ${ }^{\ddagger \ddagger}$, Adriano Pastore ${ }^{\ddagger \ddagger}$ \\ * atlanTTic Research Center, Universidade de Vigo, Galicia, Spain \\ $\dagger$ DIGCOM research group, TELIN Department, Ghent University, Ghent, Belgium \\ $\ddagger$ Centre Tecnològic de Telecomunicacions de Catalunya (CTTC), Castelldefels, Spain \\ Email: \{mosquera, tramirez\}@gts.uvigo.es, Nele.Noels@ugent.be, \{marius.caus, adriano.pastore\}@cttc.cat
}

\begin{abstract}
A novel coding strategy is proposed for a broadcast setting with two transmitter (TX) antennas and two singleantenna receivers $(\mathbf{R X})$. The strategy consists of using spacetime block coding to send a common message (to be decoded by both RXs) across the two TX antennas, while each TX antenna also sends a private message to one of the RXs. The relative weight of the private and common messages from each TX antenna is tuned to maximize the instantaneous achievable sum-rate of the channel. Closed-form expressions for the optimal weight factors are derived. In terms of the generalized degrees of freedom (GDoF) metric, the new scheme is able to achieve the sum-GDoF with finite precision channel state information at the transmitter (CSIT) of the two user broadcast channel. Moreover, as opposed to the existing rate-splitting schemes, the proposed scheme yields instantaneous achievable rates that are independent of the channel phases. This property is instrumental for link adaptation when only magnitude CSIT is available. Our numerical results indeed demonstrate the superiority of the scheme for the 2-user setting in case of magnitude CSIT. Extension to a more general $K$-user scenario is briefly discussed.
\end{abstract}

Index Terms-Achievable rates, Broadcast channel, MISO, Partial CSIT, Rate splitting.

\section{INTRODUCTION}

Multi-antenna terrestrial base stations [1] and multi-beam satellite systems [2] play a key role in both current and forthcoming high-throughput communication networks. For single-antenna terminals, both settings can be featured by the multiple-input single-output broadcast channel (MISOBC). The physical layer performance of the corresponding MISO broadcast communication system largely depends on the accuracy of the downlink channel information that is available at the transmitter (TX). In practice, however, there are limitations on the overhead that can be imposed by (i) the transmission of channel estimation training symbols, and (ii) the exchange of channel estimates between the receiver (RX) and the TX. The result is that, at best, the TX has only partial channel information at its disposal.

Under full channel state information at the transmitter (CSIT), the Shannon capacity region of the MISO-BC is achieved by means of dirty-paper coding (DPC) [3], [4]. With imperfect CSIT, finding the Shannon capacity region has proven elusive, although major steps have been taken recently by using the degrees-of-freedom (DoF) and generalized DoF (GDoF) frameworks. In particular, for partial CSIT, i.e., when the channel estimation error decays with increasing signal-tonoise ratio (SNR) at a rate of $O\left(\mathrm{SNR}^{-\beta}\right)$ with $\beta \geq 0$, [5] and [6] have proposed TX schemes that achieve the DoF and the GDoF of the MISO-BC, respectively. Here, the parameter $\beta$ captures the amount of channel uncertainty, so that higher $\beta$ denotes a knowledge of the channel which improves more rapidly with the SNR. The schemes in [5], [6] are both based on rate-splitting (RS) techniques, which rely on encoding information into private and common messages: the common message is multicast to all the RXs and placed on top of the private messages to the individual RXs. This superposition of messages is such that the RXs must first decode the common message, which is then cancelled from the received signal before the respective private messages are decoded. To the authors' knowledge, the first paper to notice the relevance of RS for the MISO-BC with partial CSIT was [7]. Therein, a mixture of imperfect current CSIT and perfect delayed CSIT is considered, and RS is seen to be applicable to those scenarios with imperfect current CSIT. Before that, RS had already been considered for the interference channel, i.e., without collaboration among the TX antennas, by Han and Kobayashi (HK) in their seminal paper [8]. Some very recent studies on the use of RS schemes in multi-cell settings have been presented in [9], [10].

DoF and GDoF formulations are optimal in the infinite SNR regime: they allow to de-emphasize the effects of additive noise [10], so that they do not necessarily lead to the best approach for finite SNR regimes. In this latter case, there is always a compromise between interference cancelling and enhancing the detectability of the desired signal in the presence of noise [7]. As an example, consider the use of space-time block coding (STBC) in [11] to improve the performance of the encoding scheme for outdated CSIT in [12] at finite SNR, or the use of an additional common message transmitted by means of a STBC in [13], thus improving the ergodic sum-rate with respect to the baseline RS scheme.

In the current paper, we are particularly interested in those scenarios for which only magnitude CSIT is available. Channel phase usually varies faster than magnitude, and may pose a significant burden if reported back to the TX [14]. It may be also the case that cooperation among different transmit antennas can be limited due to the lack of phase coherence or precise time alignment among the different radio frequency chains, so that only channel magnitude is to be entrusted for the design of the transmit scheme and joint selection of information rates. This limitation can be more commonly 
found in multibeam communication satellites [2]. We will therefore assume a channel with magnitude fixed for the duration of the codewords and perfectly known to the TX, and phase possibly non constant and completely unknown to the TX. Magnitude CSIT can be considered as a nuanced instance of partial CSIT, with $\beta=0$ (more details will be provided in Section III). When $\beta=0$, the CSIT does not improve with SNR: this case has also been termed as finite precision CSIT in the literature [6], [15]. For this magnitude CSIT model, we design and optimize a particular RS scheme. Whereas the scheme in [5] relies on precoding and the scheme in [6] simply transmits a scaled version of the same common message from both antennas, the multicast of the common message is performed by means of an STBC in our design, which is especially suited in the absence of full CSIT [16]. The main driver for this approach is the performance in the finite SNR regime. We will show that the proposed RS scheme achieves the optimum sum-GDoF at high SNR, while outperforming the existing schemes in terms of achievable sum-rate at finite SNR, under magnitude CSIT. An important practical advantage of the proposed RS scheme is that the instantaneous achievable rates are independent of the channel phases, in such a way that the knowledge of channel magnitudes suffices for the instantaneous selection of the optimal design parameter values. With respect to [17], where the authors first pointed out a similar scheme as a potential method to deal with the lack of phase information in a multibeam satellite setting, a more detailed study is performed here. In short, the main contributions which extend the initial work in [17], are: (i) a GDoF analysis is presented; (ii) the analytical derivation of optimal weights for private and common messages in the finite SNR regime is performed; (iii) a general overview of relevant schemes is addressed; (iv) practical implementation aspects are considered, such as the use of the Alamouti STBC for the multicasting of the common message or synchronization; (v) a comprehensive evaluation under different operation regimes is presented.

The paper proceeds as follows. After presenting the system model in Section II, a brief review of previous results is exposed in Section III. The operation of the proposed spacetime rate splitting (STRS) scheme is contained in Section IV for a 2-user MISO-BC and its extension to a more general $K$-user scenario is discussed in Section V. The analytical sum-rate optimization is carried out in Section VI. The GDoF performance is analyzed in Section VII. Numerical results are detailed in Section VIII to assess the relative merits of the proposed RS approach with respect to other techniques and theoretical bounds. Finally, Section IX concludes the paper.

Notation: Upper (lower) boldface letters denote matrices (vectors). (. $)^{H}$ and $\mathbf{I}_{N}$ denote Hermitian transpose and $N \times N$ identity matrix, respectively. $\mathbb{E}[\cdot]$ and $\operatorname{tr}\{\cdot\}$ are the expectation and matrix trace operators, respectively.

\section{SySTEM MOdEL}

For a generic number of users $K$, the MISO-BC is modeled as $\mathbf{y}=\mathbf{H x}+\mathbf{w}$, with $\mathbf{y} \in \mathbb{C}^{K \times 1}$ the received values at the $K$ single-antenna user terminals, $\mathbf{H} \in \mathbb{C}^{K \times K}$ the square channel matrix, $\mathbf{x} \in \mathbb{C}^{K \times 1}$ the symbol vector transmitted by the $K$ antennas, and $\mathbf{w} \in \mathbb{C}^{K \times 1}$ a vector of zero-mean unit variance additive white Gaussian noise (AWGN) samples, such that $\mathbb{E}\left[\mathbf{w} \mathbf{w}^{H}\right]=\mathbf{I}_{K}$. The TX power is given by $P=\operatorname{tr}\left\{\mathbf{x} \mathbf{x}^{H}\right\}$, and for commonly found technological constraints, the average per-antenna power will be upper bounded by $P / K$. Cooperation among the TX antennas is allowed, but there is no cooperation among the RX terminals. It is worth recalling that the column vector $\mathbf{y}$ is obtained by stacking columnwise the signal received by all users at a given time slot. For convenience and simplicity, the time index is omitted.

We consider a block-fading channel, with the magnitude of the channel entries $[\mathbf{H}]_{i, j}=h_{i, j}$ constant during the transmission of a codeword. We will focus on the two-user MISO-BC, whose system equations read as

$$
\begin{aligned}
& y_{1}=h_{1,1} x_{1}+h_{1,2} x_{2}+w_{1}, \\
& y_{2}=h_{2,1} x_{1}+h_{2,2} x_{2}+w_{2} .
\end{aligned}
$$

When applying this simplistic model it is tacitly assumed that TX antennas and RX terminals are conveniently paired for the duration of a time slot. The more general setting with an arbitrary number of users $K$ is addressed in Section V.

The components $x_{j}$ and $w_{k}$ denote the symbol transmitted by the $j$-th TX antenna and the noise sample that contaminates the $\mathrm{RX}$ of the $k$-th user terminal, respectively. Each $\mathrm{RX}$ is assumed to have full knowledge of its respective channel entries. The CSIT depends on the feedback of this information to the TX. As explained in the introduction, we are particularly interested in the case of magnitude CSIT, where the TX is oblivious to the channel phase information, but perfectly knows the channel quality of all links, i.e.,

$$
\gamma_{j, k}=\frac{P}{2}\left|h_{j, k}\right|^{2} \text {. }
$$

Note that $\gamma_{j, k}$ can be identified with the SNR (the noise power is normalized to 1 ) of the link from antenna $k$ to receiver $j$.

\section{REVIEW OF RELATED RESULTS}

The system model outlined in the previous section corresponds to a highly relevant setting with unknown capacity region. Before presenting a new, practical coding scheme for this setting, with its corresponding achievable rate region, we review some related results.

\section{A. MISO-BC with partial CSIT}

The magnitude CSIT scenario can be considered as a nuanced instance of the finite precision CSIT model studied, for example, in [15]:

$$
h_{i, j}=\sqrt{P^{\alpha_{i, j}}} G_{i, j} .
$$

Under the finite precision CSIT model (4), TXs are assumed to be aware of the $\alpha_{i, j}$ values, i.e., the coarse channel strength parameters, but not the precise $G_{i, j}$ values. For the $G_{i, j}$, the TXs are only aware of the joint probability density function. One of the basic assumptions in the works on the GDoF of the MISO-BC is that the density functions of the channel entries $h_{i, j}$ are bounded, so that zero measure spaces 
have a zero probability $[18$, Ch. 2]. A random phase error distribution, for example uniform in the range $[0,2 \pi)$, together with known magnitude for the channel coefficients, would yield an unbounded density function or, in other words, the support for the unknown channel coefficients would have zero measure; this seems to exclude magnitude CSIT from the finite precision paradigm, although an arbitrarily small uncertainty in the magnitude of the coefficients would suffice to overcome this limitation.

The finite precision CSIT model (4) is, in fact, a special case of the partial CSIT model considered in [5] and related works:

$$
h_{i, j}=\hat{h}_{i, j}+\tilde{h}_{i, j},
$$

with $\hat{h}_{i, j}$ the channel estimates, and $\tilde{h}_{i, j}$ the zero-mean random estimation error terms with variance $\mathbb{E}\left[\left|\tilde{h}_{i, j}\right|^{2}\right]=P^{-\beta}$. The accuracy of the CSIT is parameterized by the quantity $\beta$, ranging from perfect $\operatorname{CSIT}(\beta=\infty)$ till finite precision CSIT $(\beta=0)^{1}$. In the magnitude CSIT model, considered in the current paper, the uncertainty about the channel does not decrease with the SNR. It can therefore be assimilated to the finite precision case $(\beta=0)$ to a high degree.

In [15], a so-called interference enhancement (ENH) scheme was shown to achieve the GDoF of the MISO-BC under finite precision CSIT. The setting in [15] considers a global power constraint, but a straightforward translation of ENH to a perantenna power constraint reads as:

$$
x_{i}=\sqrt{P\left(1-\lambda_{i}\right)} x_{c}+\sqrt{P \lambda_{i}} x_{1, p}, \quad i=1,2,
$$

where $x_{i}$ is the symbol transmitted from antenna $i, \lambda_{i}$ is a weight factor describing the portion of the per-antenna power allocated to the private symbol $x_{i, p}$ and $x_{c}$ denotes a common symbol. The model was generalized in [6], [18] to address the overarching partial CSIT framework. It is worth mentioning that [6], [15], [18] do not deal with actual rates at practical SNR regimes.

On the other side, [5] and related works present also a general model, aiming at the partial CSIT case and DoF performance. The transmitted symbol vector $\mathbf{x}$ is written as:

$$
\mathbf{x}=\mathbf{p}_{c} x_{c}+\mathbf{p}_{1} x_{1, p}+\mathbf{p}_{2} x_{2, p}
$$

where $\mathbf{p}_{c}, \mathbf{p}_{1}$ and $\mathbf{p}_{2}$ are precoding vectors of a common symbol $x_{c}$ and of two private symbols $x_{1, p}$ and $x_{2, p}$, respectively. This scheme has been shown to achieve the DoF optimality.

Perhaps the most important difference between the schemes from (6) and (7) and the scheme considered in this paper is that in (6) as well as in (7) the same common symbol is transmitted from both antennas. As a result, the instantaneous achievable rates depend on the instantaneous values of the channel phases

\footnotetext{
${ }^{1}$ A large number of practical settings can be categorized as finite precision CSIT, since a fixed amount of resources is reserved for signaling in the reverse link in frequency-division duplexing (FDD) schemes, so that the quality of the CSIT is independent of the SNR. However, the quality of the CSIT could be dependent on the link SNR if, for instance, time-division duplexing (TDD) is used, so that the channel is estimated at the transmitter based on the reverse link pilots, as long as the SNR of the uplink and downlink is highly correlated. More in general, if $\beta \log P$ quantization bits are employed per scalar, then a mean squared error $P^{-\beta}$ can be achieved [19], giving rise to a model such as (5).
}

which are not available at the TX in the case of magnitude CSIT. This implies that actual rates cannot be used for the design of adaptation mechanisms. As a work-around, ergodic or worst case rates are the metrics considered in [5], [20].

\section{B. MISO-BC with vector magnitude CSIT}

In some sense, the considered MISO BC magnitude CSIT model is affiliated to the scenario of a vector broadcast channel with perfect channel state information at the receiver and vector channel magnitude information at the TX, studied in [21]. In this case the RXs report the total received power, which amounts to the TX having access to the norm of the different rows of the channel matrix $\mathbf{H}$. It has been proved in [21] that the capacity region is the same as that for the scalar broadcast case, and it is achieved by superposition coding (SC) and successive decoding. From [21, Lemma 5], the transmit vector $\mathbf{x}=\mathbf{u}_{1}+\ldots+\mathbf{u}_{K}$ is the superposition of $K$ independent jointly Gaussian vectors, each following $\mathbf{u}_{k} \sim \mathcal{C} \mathcal{N}\left(0, \mathbf{I}_{K} P_{k} / K\right)$, and $P_{k}$ the power assigned to the $k$ th user. For the two-user case, we have $P_{1}=\lambda \frac{P}{2}$ and $P_{2}=(1-\lambda) \frac{P}{2}$, with $\lambda$ the relative power assigned to user 1. The boundary of the corresponding capacity region will set an inner bound for our scheme, which can exploit the additional information provided by the individual magnitude of the different links. The knowledge of the weight of individual paths provides some valuable information for the rate splitting, mostly relevant in directional settings, with significant differences in the magnitudes received at each point from each antenna.

Also related to the MISO BC under study is the MISO channel with one RX terminal and per-antenna power constraints; this case is analyzed in [22], including also the lack of CSIT.

\section{MISO-IC: no antenna cooperation}

The same $2 \times 2$ MISO setting as considered here, but without antenna cooperation is known as the MISO interference channel (IC). The capacity region of this IC is also unknown, although the Han-Kobayashi (HK) achievable rate [8] is a wellknown inner bound [23]. Similar to the scheme from [17] envisaged in this paper (see Section IV), the HK scheme is based on the splitting of the messages for users 1 and 2 into a private part (to be decoded only by the intended RX) and a common part (to be decoded by both RXs). At sender $j$, with $j \in\{1,2\}$, a fraction $\lambda_{j}$ of the power is allocated to the private part of the message addressed to user $j$, with a fraction $1-\lambda_{j}$ remaining for the common message part. As opposed to (6) (and also as opposed to the scheme from [17], which is further developed in the next section), the antennas send independent common message parts. At each RX terminal both common message parts are assumed to be decoded prior to the private message. Optimum values of the power splitting variables $\lambda_{1}$ and $\lambda_{2}$ that maximize the HK achievable sum-rate have been found in [24]-[26].

\section{Space-Time Rate Splitting}

The scheme proposed in this paper, which will be termed as space-time rate splitting (STRS), applies a rate splitting 


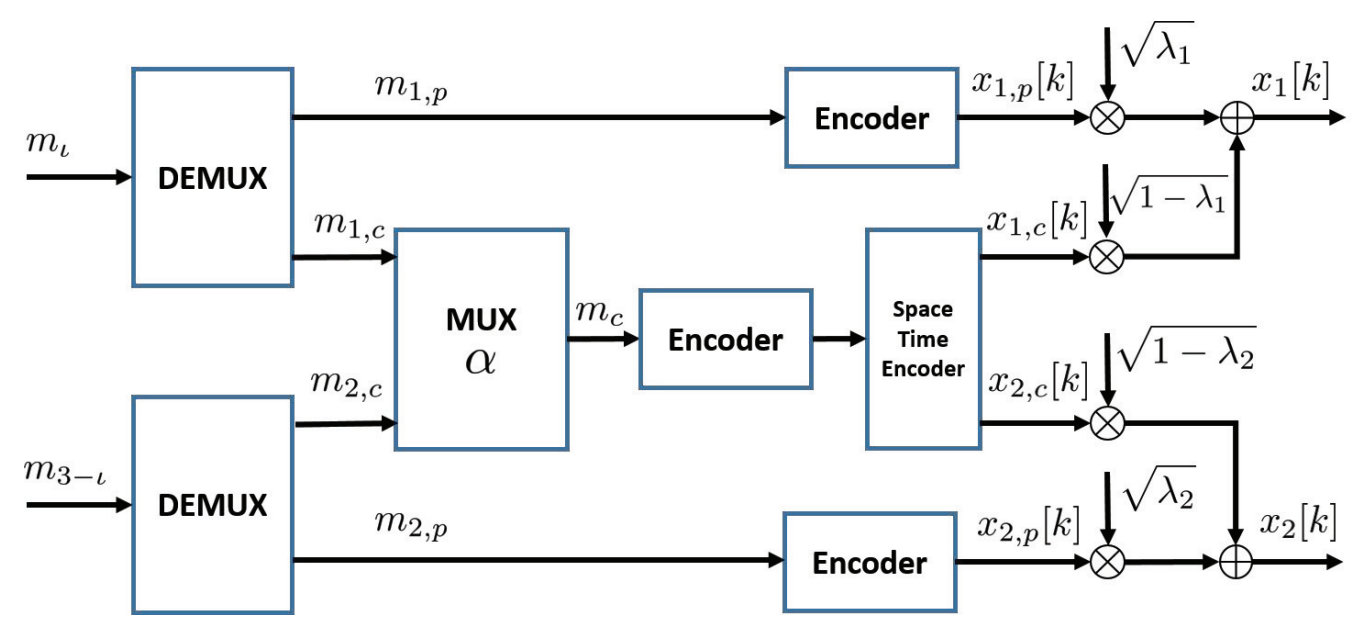

Fig. 1: Space-time rate splitting (STRS) scheme. DEMUX (MUX) denotes splitting (combining) of the incoming message(s). Message $\iota \in\{1,2\}$ is intended for user $\iota$, although for generality purposes, it may be the case that its private part is sent from antenna $3-\iota$. The parameter $\alpha$ denotes how much of the common message rate corresponds to the user $\iota$, as denoted by (10).

construction, and allows simple analysis and operation with magnitude-only CSIT. A full description of the STRS message encoding process for a two-user setting is provided in Fig. 1.

The messages $\left(m_{1}, m_{2}\right)$ intended for RXs 1 and 2, respectively, are both split into a private message part and a common message part. The private message parts are sent from only one TX antenna and are to be decoded by only one RX. We will use $m_{1, p}$ and $m_{2, p}$ to indicate the private messages sent from TX antennas 1 and 2, respectively. Depending on the channel conditions, it may be more favorable to transmit the private message to be decoded by RX 1 from TX antenna 1 than from TX antenna 2, or vice versa (See Section VI). Thus, in Fig. 1 , the message $m_{\iota}$ is split as $m_{\iota}=\left(m_{1, p}, m_{1, c}\right)$, while the message $m_{3-\iota}$ is split as $m_{\iota}=\left(m_{2, p}, m_{2, c}\right)$, with $\iota \in 1,2$. The common message $m_{c}=\left(m_{1, c}, m_{2, c}\right)$, to be decoded by both RX, is jointly sent through both antennas.

The messages $m_{1, p}$ and $m_{2, p}$ are encoded into the sequences $x_{1, p}[k]$ and $x_{2, p}[k]$, whereas the common message $m_{c}$ is encoded and mapped into $\left[x_{1, c}[k], x_{2, c}[k]\right]$ through appropriate Space-Time coding. They are subsequently transmitted by the sequences $x_{1}[k]$ and $x_{2}[k]$ as sketched in Fig. 1. For a given time instant $k, x_{1}[k]$ and $x_{2}[k]$ read as:

$$
\begin{aligned}
& x_{1}[k]=\sqrt{\frac{P}{2}\left(1-\lambda_{1}\right)} x_{1, c}[k]+\sqrt{\frac{P}{2} \lambda_{1}} x_{1, p}[k], \\
& x_{2}[k]=\sqrt{\frac{P}{2}\left(1-\lambda_{2}\right)} x_{2, c}[k]+\sqrt{\frac{P}{2} \lambda_{2}} x_{2, p}[k],
\end{aligned}
$$

where $0 \leq \lambda_{1} \leq 1$ and $0 \leq \lambda_{2} \leq 1$ denote the relative power allocated to the first and second private messages, respectively.

The splitting of the common message between the two users is regulated by a parameter $0 \leq \alpha \leq 1$, in such a way that the information rates of the two users can be expressed as

$$
\begin{aligned}
& R_{\iota}=R_{1, p}+\alpha \cdot R_{c}, \\
& R_{3-\iota}=R_{2, p}+(1-\alpha) \cdot R_{c},
\end{aligned}
$$

with $\iota \in\{1,2\}$ the index of the RX that decodes the private message $m_{1, p}$ from antenna 1 . In (10)-(11), $R_{1, p}, R_{2, p}$ and $R_{c}$ denote the information rates of the private message decoded by user 1 , the private message decoded by user 2 and the common message, respectively. The value of $\alpha$ quantifies the portion of the common message intended for $\mathrm{RX} \iota$ (i.e., the RX decoding $m_{1, p}$ ).

At the RX side, each RX first decodes the common message, cancels it from the received signal and then decodes its respective private message. Note that the private message is only communicated via the direct link between the TX antenna and the intended RX, while the common message is communicated partly via the direct link and partly via the leakage link ${ }^{2}$. The particular cases where $\left(\lambda_{1}, \lambda_{2}, \alpha, \iota\right)$ equals $(1,0,0,1),(1,0,1,2),(0,1,1,1)$ and $(0,1,0,2)$ correspond to nonorthogonal multiple access (NOMA); i.e., one of the antennas transmits a message to be decoded only by the corresponding $\mathrm{RX}$, whereas the other transmits the message intended for the other RX, and decodable by both RXs. The fact that NOMA is a subset of rate-splitting has been investigated and reported previously in works such as [27], [28].

If all messages are encoded into Gaussian codebooks, then, the achievable rate region of STRS is the convex hull of the regions $\mathcal{R}\left(\lambda_{1}, \lambda_{2}, \alpha, \iota\right)$ for $0 \leq \lambda_{1}, \lambda_{2}, \alpha \leq 1$ and $\iota \in\{1,2\}$, and with

$$
\begin{aligned}
\mathcal{R}\left(\lambda_{1}, \lambda_{2}, \alpha, \iota\right)=\left\{\left(R_{1}, R_{2}\right):\right. & R_{\iota} \leq R_{1, p}+\alpha R_{c} \\
& \left.R_{3-\iota} \leq R_{2, p}+(1-\alpha) R_{c}\right\}
\end{aligned}
$$

where

$$
\begin{gathered}
R_{1, p}=\log _{2}\left(1+\frac{\lambda_{1} \gamma_{\iota, 1}}{1+\lambda_{2} \gamma_{\iota, 2}}\right) \\
R_{2, p}=\log _{2}\left(1+\frac{\lambda_{2} \gamma_{3-\iota, 2}}{1+\lambda_{1} \gamma_{3-\iota, 1}}\right),
\end{gathered}
$$

\footnotetext{
${ }^{2}$ An extension of STRS would support the transmission of private information from both antennas to the same user by relaxing the need for decoding the common message by both RXs (message is not common anymore). This version would allow to extend slightly the rate region at the corners, when most of the rate is allocated to one user, and will not be considered in this work.
} 


$$
R_{c}=\min _{j \in\{1,2\}} \log _{2}\left(1+\frac{\left(1-\lambda_{1}\right) \gamma_{j, 1}+\left(1-\lambda_{2}\right) \gamma_{j, 2}}{1+\lambda_{1} \gamma_{j, 1}+\lambda_{2} \gamma_{j, 2}}\right)
$$

and where the quantities $\gamma_{j, k}$ are defined in (3). The common message is decoded by both terminals. Its achievable rate is set by the most restrictive terminal under the presence of the private messages. This is why the minimum of the rate of two $2 \times 1$ links needs to be taken in (14). Given the lack of phase information, the TX is unable to send symbols from both antennas which can be coherently combined at the RX terminals. The multicast rate of the common message in (14) can be achieved with Alamouti encoding, which transforms the vector channel into a scalar channel. Thus, scalar codes can be used to attain the no CSIT MISO channel capacity for the two-antenna case [21]. The respective private rates $R_{1, p}$ and $R_{2, p}$ in (12)-(13) are limited only by the interference caused by the private symbols addressed to the other user, by assuming that the common message is perfectly cancelled from the observation prior to the private message decoding.

For a given set of $\left(\lambda_{1}, \lambda_{2}, \iota\right)$ values, all rate pairs $\left(R_{1}, R_{2}\right)$ with $R_{\iota}$ given by (10) and $R_{3-\iota}$ given by (11) are achievable. The value of $\alpha$ can be changed across time without increasing the complexity of the TX and RX architectures. This allows for a seamless rate allocation which can be tailored to the users' demands while avoiding time-multiplexing of encoding schemes and the corresponding synchronization needs at the RXs. Note that for $\alpha=0$, the common message is entirely devoted to user $3-\iota$, with $R_{3-\iota}=R_{2, p}+R_{c}$ and $R_{\iota}=R_{1, p}$, whereas for $\alpha=1$, the opposite happens, and $R_{\iota}=R_{1, p}+R_{c}$ and $R_{3-\iota}=R_{2, p}$. Maximum fairness, in the sense that $R_{\iota}=$ $R_{3-\iota}$ (without having to use time-sharing) is only possible if

$$
R_{c} \geq\left|R_{1, p}-R_{2, p}\right|
$$

If $R_{c}$ is strictly smaller than $\left|R_{1, p}-R_{2, p}\right|$, then $R_{\iota}$ is strictly larger (smaller) than $R_{3-\iota}$ for any $\alpha \in[0,1]$, if $R_{1, p}$ is smaller (larger) than $R_{2, p}$. If (15) holds, then $R_{1}=R_{2}$ is achieved for $\alpha=\left(R_{2, p}-R_{1, p}+R_{c}\right) /\left(2 R_{c}\right)$. It is important to note that the STRS sum-rate $R_{\text {sum }}^{\mathrm{STRS}}=R_{1, p}+R_{2, p}+R_{c}$ does not change with $\alpha$, and that the condition (15) does not depend on $\alpha$, nor on $\iota$.

As exposed earlier, STRS uses less information than DPC and other linear precoding schemes (e.g., [5] and related works) at the TX side. In addition, thanks to the use of the Alamouti scheme, both users' contributions to the common message are easily decoupled at the RX. This makes decoding simpler than HK's joint decoding at the RX terminals [8]. Finally, actual rates can be used as design metrics by STRS, since they are independent of the channel phase (which is often not accurately known at the TX). As it turns out, analytical expressions of the optimal weights of the private and the common contributions in STRS can be derived in closed-form as a function of magnitude CSIT only (see Section VI). These features make STRS a scheme amenable for practical use.

\section{EXTENSION TO $K$ USERS}

In the previous section, we have described the STRS scheme for a two-user MISO BC. Next, we sketch the structure of the
STRS scheme for $K$ users. In the $K$-user setting, public messages addressed to different groups of users need to be devised along the line of [29]-[31]. For the three-user case, [30] proves that a hierarchy of public messages is needed to achieve the GDoF in the MISO BC with finite precision CSIT. In [29] and [31] a multi-layer RS scheme is presented, which requires a number of successive interference cancellation stages at the RXs equal to the number of layers of common messages. Each user message is partitioned into several independent submessages, which are to be decoded by different subsets of users which include the intended destination of the original message.

For illustration purposes, let us consider the case $K=3$, and the message $m_{1}$ addressed to the first user in Section IV. This message should be split and encoded into $x_{123, c}, x_{12, c}$, $x_{13, c}$ and $x_{p, 1}$, which are intended for all users, for users $(1,2)$, for users $(1,3)$, and for user 1 , respectively. Thus, the signal transmitted by antenna 1 can be expressed as:

$$
\begin{aligned}
x_{1}= & \sqrt{\left(1-\lambda_{1}\right) P} x_{123, c}+\sqrt{\lambda_{1}\left(1-\lambda_{2}\right) P} x_{12, c} \\
& +\sqrt{\lambda_{1} \lambda_{2}\left(1-\lambda_{3}\right) P} x_{23, c}+\sqrt{\lambda_{1} \lambda_{2} \lambda_{3}\left(1-\lambda_{4}\right) P} x_{13, c} \\
& +\sqrt{\lambda_{1} \lambda_{2} \lambda_{3} \lambda_{4} P} x_{p, 1}, 0 \leq \lambda_{i} \leq 1, i=1,2,3,4 .
\end{aligned}
$$

The number of weight factors has increased to four, which entails a higher optimization complexity.

More in general, a message $m_{k}$ is split into different submessages $m_{\mathcal{K}_{i}, c}$, which are sub-messages to be decoded by the users which are contained in the set $\mathcal{K}_{i}$. The cardinality of $\mathcal{S}_{k}=\bigcup_{i} \mathcal{K}_{i}$ is the number of all possible combinations of the users which include user $k$. A detailed analysis of the grouping for RS schemes is presented in [31]. The number of SIC stages at the RXs follow from the depth of the grouping subsets, i.e., the number of subsets that a given user belongs to. The contribution of some common streams is expected to be marginal, so they can be removed without much impact [31]. As a result, two-layer grouping is quite a common strategy, with a common message addressed to all users, followed by different sub-messages intended for a second layer of $G$ disjoint subsets $\mathcal{K}_{g}, g=1, \ldots, G$; this is the approach followed, for example, in [29] and [32], which requires only two SIC stages at the RXs. If, for simplicity, and without loss of generality, we assume that $\mathrm{RX} i$ decodes the private message from TX $i$, then the achievable rates with $K$ users under this two-layer grouping can be obtained similarly to those for the two-user case in (12)-(14). Each user must decode first the rate $R_{c}$ message common to all users, then the rate $R_{g, c}$ common message to the group $\mathcal{K}_{g}$ that the user happens to belong to, and then its rate $R_{i, p}$ private message:

$$
\begin{gathered}
R_{c}=\min _{j \in\{1, \ldots, K\}} \log _{2}\left(1+\frac{\sum_{i}\left(1-\lambda_{i, 1}\right) \gamma_{j, i}}{1+\sum_{i} \lambda_{i, 1} \gamma_{j, i}}\right), \\
R_{g, c}=\min _{j \in \mathcal{K}_{g}} \log _{2}\left(1+\frac{\sum_{i \in \mathcal{K}_{g}} \lambda_{i, 1}\left(1-\lambda_{i, 2}\right) \gamma_{j, i}}{1+\sum_{i \in \mathcal{K}_{g}} \Gamma_{i, j}+\sum_{i \notin \mathcal{K}_{g}} \lambda_{i, 1} \gamma_{j, i}}\right), \\
R_{i, p}=\log _{2}\left(1+\frac{\lambda_{i, 1} \lambda_{i, 2} \gamma_{i, i}}{1+\sum_{j \in \mathcal{K}_{g}, j \neq i} \Gamma_{j, i}+\sum_{j \notin \mathcal{K}_{g}} \lambda_{j, 1} \gamma_{i, j}}\right),
\end{gathered}
$$


with $\Gamma_{i, j}=\lambda_{i, 1} \lambda_{i, 2} \gamma_{j, i}$. Note that each antenna $i$ transmits messages with relative power $1-\lambda_{i, 1}, \lambda_{i, 1}\left(1-\lambda_{i, 2}\right)$ and $\lambda_{i, 1} \lambda_{i, 2}$ for the global common message, the message common to the group at which user $i$ belongs to, and its private message, respectively. The achievable rate region is the convex hull of the regions $\mathcal{R}\left(\lambda_{i, 1}, \lambda_{i, 2}, \alpha_{i}, \beta_{i}\right)$ for $0 \leq \lambda_{i, 1}, \lambda_{i, 2} \leq 1$, $\sum_{i \in \mathcal{K}_{g}} \alpha_{i} \leq 1, \sum_{i=1}^{K} \beta_{i}=1$ and $i \in\{1, \ldots, K\}$, with

$$
\begin{aligned}
\mathcal{R}\left(\lambda_{i, 1}, \lambda_{i, 2}, \alpha_{i}, \beta_{i}\right)=\left\{\left(R_{i}, i=1, \ldots, K\right):\right. & R_{i} \leq R_{i, p}+ \\
& \left.\alpha_{i} R_{g, c}+\beta_{i} R_{c}\right\} .
\end{aligned}
$$

Finally, note that no full-rate orthogonal space-time code exists for the $K>2$ case with complex symbols. Full-rate spacetime codes sacrifice the orthogonality for $K>2$ and, in consequence, the ability to use symbol-wise decoding of the space-time encoded common message, with increased receiver complexity as a result [33].

\section{Achievable Sum-Rate Maximization}

In STRS, the knowledge of the channel quality information can be exploited to optimally split the power over the different sub-messages. As observed in the previous section, the number of parameters grows linearly with the number of users in the best case; for example, for a two-layer grouping strategy, with one global common message and a second layer of submessages addressed to the different disjoint subsets, on top of the private messages, the number of parameters is $2 K$ in most cases. Thus, the complexity of the optimization increases significantly as compared to the sub-optimal approach where TX antenna pairs and user pairs are combined and modelled as separate two-user MISO BCs: one $2 K$-dimensional optimization, as compared to $K / 2$ two-dimensional optimizations. In consequence, we will address the maximization of the sumrate for the $K=2$ case.

In the following, we derive analytical expressions ${ }^{3}$ for the values of $\iota, \lambda_{1}$ and $\lambda_{2}$ for which the sum-rate, i.e.,

$$
R_{\text {sum }}^{\mathrm{STRS}}=R_{1, p}+R_{2, p}+R_{c}
$$

achieves a maximum. We will denote this maximum as $\max \left(R_{\text {sum }}^{\mathrm{STRS}}\right)$. The obtained expressions are instrumental for practical system design and instantaneous rate adaptation. Moreover, they will serve, in Section VII, to show that STRS is optimal at high SNR, in the sense that it can achieve the maximum sum-GDoF of the considered 2-user MISO BC with magnitude CSIT.

We recall that $\iota \in\{1,2\}$ denotes the index of the $\mathrm{RX}$ that decodes the private message $m_{1, p}$ from TX antenna 1. Now, if we define the quantities $r_{\iota ; a}$ and $r_{\iota ; b}$, as

$$
\begin{aligned}
& r_{\iota ; a}\left(\lambda_{1}, \lambda_{2}\right)=\log _{2}\left(\frac{\left(1+\lambda_{1} \gamma_{1,1}+\lambda_{2} \gamma_{1,2}\right)\left(1+\gamma_{2,1}+\gamma_{2,2}\right)}{\left(1+\lambda_{1} \gamma_{3-\iota, 1}\right)\left(1+\lambda_{2} \gamma_{\iota, 2}\right)}\right), \\
& r_{\iota ; b}\left(\lambda_{1}, \lambda_{2}\right)=\log _{2}\left(\frac{\left(1+\lambda_{1} \gamma_{2,1}+\lambda_{2} \gamma_{2,2}\right)\left(1+\gamma_{1,1}+\gamma_{1,2}\right)}{\left(1+\lambda_{1} \gamma_{3-\iota, 1}\right)\left(1+\lambda_{2} \gamma_{\iota, 2}\right)}\right),
\end{aligned}
$$

\footnotetext{
${ }^{3}$ In terms of the channel link qualities $\left\{\gamma_{j, k}\right\}$ from (3).
}

it can be readily checked from (12)-(14) and (20) that, for given $\iota$,

$$
R_{\text {sum }}^{\mathrm{STRS}}=\left\{\begin{array}{ll}
r_{\iota ; a} & , \beta_{1}\left(1-\lambda_{1}\right) \geq \beta_{2}\left(1-\lambda_{2}\right) \\
r_{\iota ; b} & , \beta_{1}\left(1-\lambda_{1}\right) \leq \beta_{2}\left(1-\lambda_{2}\right)
\end{array},\right.
$$

with $\beta_{j}$, for $j \in\{1,2\}$, given by

$$
\beta_{j}=\gamma_{j, j}-\gamma_{3-j, j}-\gamma_{j, 3-j} \gamma_{3-j, j}+\gamma_{j, j} \gamma_{3-j, 3-j} .
$$

We recall that the quantities $\lambda_{i}$ in (21)-(23) denote the relative weight of the private message component in the signal from TX antenna $i$. We also recall that the quantities $\gamma_{j, k}$ in (21)(22) and (24) denote the channel quality of the link from TX antenna $k$ to $\mathrm{RX} j$.

A partition of the $\left(\lambda_{1}, \lambda_{2}\right)$ domain

$$
0 \leq \lambda_{1} \leq 1, \quad 0 \leq \lambda_{2} \leq 1
$$

results from (23). This partition is illustrated in Fig. 2, where the left-hand plot corresponds to $\iota=1$ and the right-hand plot applies to $\iota=2$. The points labelled $E$ and $F$ in Fig. 2 have coordinates

$$
E:(1,1) \text { and } F:\left(0,1-\beta_{1} / \beta_{2}\right) .
$$

Above the line segment $[E F]$ in Fig. 2, the sum-rate equals (21). Below it, the sum-rate equals (22). And for $\left(\lambda_{1}, \lambda_{2}\right)$ along $[E F]$, we have

$$
\begin{aligned}
R_{\text {sum }}^{\mathrm{STRS}} & =r_{1 ; a}\left(\lambda_{1}, 1-\frac{\beta_{1}}{\beta_{2}}\left(1-\lambda_{1}\right)\right) \\
& =r_{1 ; b}\left(\lambda_{1}, 1-\frac{\beta_{1}}{\beta_{2}}\left(1-\lambda_{1}\right)\right) \equiv r_{[E F]}\left(\lambda_{1}\right) .
\end{aligned}
$$

Fig. 2 also contains arrows indicating the direction in which

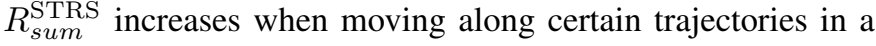
specific part of the domain. From (21)-(22), it can indeed be verified that, for (25),

(P1) $r_{1 ; b}$ and $r_{2 ; a}$ are non-increasing functions of $\lambda_{1}$, for a fixed $\lambda_{2}$.

(P2) $r_{1 ; a}$ and $r_{2 ; b}$ are non-increasing functions of $\lambda_{2}$, for a fixed $\lambda_{1}$.

(P3) $r_{1 ; b}$ and $-r_{2 ; a}$ are increasing (resp. decreasing, resp. constant) functions of $\lambda_{2}$, for $\lambda_{1}$ equal to zero, if $\gamma_{2,2}$ is larger than (resp. smaller than, resp. equal to $\gamma_{1,2}$ ).

(P4) $r_{1 ; a}$ and $-r_{2 ; b}$ are increasing (resp. decreasing, resp. constant) functions of $\lambda_{1}$, for $\lambda_{2}$ equal to zero, if $\gamma_{1,1}$ is larger than (resp. smaller than, resp. equal to $\gamma_{2,1}$ ).

Without loss of generality, we assume that the numbering of the RXs and the TX antennas is such that

(I1) $\gamma_{2,2} \geq \gamma_{1,2}$.

(I2) $\gamma_{2,2}-\gamma_{1,2} \geq \gamma_{1,1}-\gamma_{2,1}$.

(I3) $\left(1+\gamma_{2,2}\right)\left(1+\gamma_{1,1}\right) \geq\left(1+\gamma_{1,2}\right)\left(1+\gamma_{2,1}\right)$.

Thus, it is easily verified that the $\lambda_{2}$-coordinate of the point $F$ in Fig. 2 (see (26)) is always positive-valued (although it can rise above 1 , for $\beta_{1}<0$ ).

To derive the maximum STRS sum-rate and the corresponding parameter values, we now follow the steps in Table I. First, we determine the $\left(\lambda_{1}, \lambda_{2}\right)$ pair that yields the highest sum-rate $R_{\text {sum }}^{\text {STRS }}$ (23) for fixed $\iota$, with $\iota=1$ and $\iota=2$. To do so, we rely on (I1)-(I3) and (P1)-(P4) with graphical illustration in 

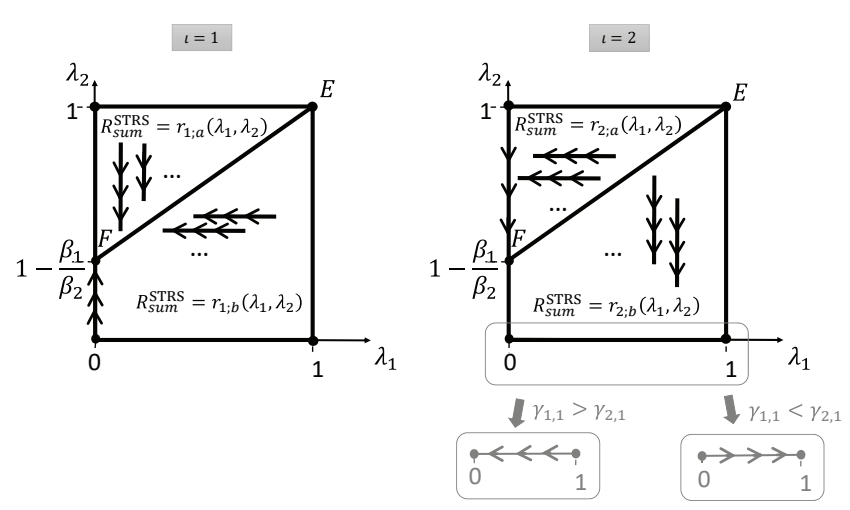

Fig. 2: Partitioning of the power splitting domain $\left(\lambda_{1}, \lambda_{2}\right) \in$ $[0,1]^{2}$ and appropriate STRS sum-rate expressions, according to (23). The left- and right-hand plots correspond to $\iota=1$ and $\iota=2$, respectively. Arrows indicate directions of increasing STRS sum-rate $R_{\text {sum }}^{\mathrm{STRS}}$.

Fig. 2. It follows that, for given $\left(\gamma_{1,1}, \gamma_{2,2}, \gamma_{1,2}\right)$ with (I1), five regimes emerge depending on the value of $\gamma_{2,1}$ :

$$
\begin{aligned}
\max \left(0, \gamma_{1,1}-A\right) \stackrel{(\mathrm{I} 2)}{\leq} \gamma_{2,1} & \leq \frac{B+A}{B+A\left(1+\gamma_{1,2}\right)} \gamma_{1,1} \\
\frac{B+A}{B+A\left(1+\gamma_{1,2}\right)} \gamma_{1,1}<\gamma_{2,1} & \leq \gamma_{1,1} \\
\gamma_{1,1}<\gamma_{2,1} & \leq \frac{1+\gamma_{2,2}}{1+\gamma_{1,2}} \gamma_{1,1} . \\
\frac{1+\gamma_{2,2}}{1+\gamma_{1,2}} \gamma_{1,1}<\gamma_{2,1} & \stackrel{(\mathrm{I} 3)}{\leq} \frac{1+\gamma_{2,2}}{1+\gamma_{1,2}} \gamma_{1,1}+\frac{A}{1+\gamma_{1,2}}
\end{aligned}
$$

with $A=\gamma_{2,2}-\gamma_{1,2}, B=\gamma_{1,1} \gamma_{2,2}$.

The left-hand side of Fig. 2 learns that, if $\iota=1$, maximum sum-rate is achieved in:

- The point on the line segment $[E F]$ in Fig. 2 where (27) is maximum, if (28)-(30). We will refer to the $\lambda_{1}$-coordinate of this point as $\lambda_{1}^{\star}$ (see (32)-(33)).

- The point with coordinates $\left(\lambda_{1}, \lambda_{2}\right)=(0,1)$, if (31).

It follows from (27) that

$$
\lambda_{1}^{\star}= \begin{cases}\lambda^{\star} & , \text { if }(28) \\ 0 & , \text { otherwise }\end{cases}
$$

where

$\lambda^{\star}=\min \left\{\frac{\gamma_{1,2}-\gamma_{2,2}+\sqrt{\frac{\gamma_{1,1} \gamma_{2,2}\left(\gamma_{1,1}-\gamma_{2,1}\right)\left(\gamma_{2,2}-\gamma_{1,2}\right) \beta_{2}}{\gamma_{1,2} \gamma_{2,1} \beta_{1}}}}{\gamma_{1,1} \gamma_{2,2}-\gamma_{1,2} \gamma_{2,1}}, 1\right\}$.

For $\iota=2$, it follows, from the right-hand side of Fig. 2, that maximum sum-rate is achieved in

- The point with coordinates $\left(\lambda_{1}, \lambda_{2}\right)=(0,0)$, if (28)-(29).

- The point with coordinates $\left(\lambda_{1}, \lambda_{2}\right)=(1,0)$, if (30)-(31).

These results and the expressions for the corresponding sumrate maxima for given $\iota$ are summarized in lines 1-4 of Table I.
Then, we determine the value of $\iota$ that yields the highest sumrate. Taking into account (I1)-(I3) and (P1)-(P4), it can be verified that, for all $\gamma_{2,1}$ (28)-(31), a higher maximum sumrate is achieved for $\iota=1$ than for $\iota=2$. The overall maximum sum-rate, denoted as $\max \left(R_{\text {sum }}^{\mathrm{STRS}}\right)$, and the corresponding parameter values are summarized in lines 5-6 of Table I.

From Table I, (24), (27), (33) and (I1)-(I3), we make the following observations.

1) For values of $\gamma_{2,1}$ that are small enough for (28) to hold, there is an optimum way to distribute the TX antenna power between the common and the private messages. In this optimally configured system, the power allocation between common and private messages is such that the signal-to-noise-plus-interference ratio for the common message is the same at both RXs, so that the minimum in (14) is equal to both its arguments. To accomplish this, TX antenna 1 needs to allocate more power to the common message than TX antenna 2; this is immediate consequence of assumption (I2).

2) The conditions in (28) are typically met in scenarios where the direct link SNRs are (a) substantially larger than $0 \mathrm{~dB}$, and (b) large as compared to the leakage link SNRs. In this case, the optimal weight factor is more or less the same for both antennas, and approximately given by:

$$
\lambda_{1}^{\star} \approx \lambda_{2}^{\star} \approx \min \left\{\left(\sqrt{\gamma_{1,2} \gamma_{2,1}}\right)^{-1}, 1\right\} .
$$

It follows that the amount of power that is allocated to the common message is mainly determined by the SNRs of the leakage links, and tends to increase as either of the leakage link SNRs increases. If the leakage link SNRs themselves are also considerably larger than $0 \mathrm{~dB}$, the optimum weight factor (34) is significantly smaller than 1.

3) For values of $\gamma_{2,1}$ that are large enough for (31) to hold, it is recommended that TX antenna 1 allocates all of its power to the common message, while TX antenna 2 allocates its power entirely to a private message to $\mathrm{RX}$ 2. This situation occurs if, irrespective of the TX power allocation, the multicast rate of the common message (14) is always limited by the quality of the weaker of the two $2 \times 1$ user links (i.e., the link to RX 1 ).

4) For intermediate values of $\gamma_{2,1}$, for which either (29) or (30) applies, it is optimum for TX antenna 1 to allocate all of its power to the common message, while for TX antenna 2 there is still an optimum way to allocate its power between the common and the private messages. The corresponding optimal weight factor $\lambda_{2}$ is such that, if TX antenna 1 only transmits a common message, the signal-to-noise-plus-interference ratio for the common message is the same at both RXs. It is easily seen that this $\lambda_{2}$ is an increasing function of $\gamma_{2,1}$, with $\lambda_{2}$ going to 1 for $\gamma_{2,1}$ approaching the upper bound in (30).

As a concluding remark, we recall that STRS, in general, and therefore sum-rate optimal STRS, in particular, does not necessarily result in a fair distribution of the rates among the users. Equal user rates, for example, can only be achieved if the condition (15) is met. For a given set of link SNR values, 


\begin{tabular}{|c|c|c|c|c|}
\hline \multicolumn{2}{|l|}{$\gamma_{2,1}:$} & $(31)$ & $(29)$ & $(28)$ \\
\hline \multirow{2}{*}{$\begin{array}{l}\left(\lambda_{1}, \lambda_{2}\right) \text { yielding largest } \\
R_{\text {sum }}^{\mathrm{STRS}}, \text { for given } \iota\end{array}$} & $\iota=1$ & $(0,1)$ & $\left(0,1-\frac{\beta_{1}}{\beta_{2}}\right),(24)$ & $\left(\lambda^{\star}, 1-\frac{\beta_{1}}{\beta_{2}}\left(1-\lambda^{\star}\right)\right),(24),(33)$ \\
\hline & $\iota=2$ & \multirow{2}{*}{\multicolumn{2}{|c|}{$\begin{array}{r}(1,0) \\
(0,1)(20)\end{array}$}} & $(0,0)$ \\
\hline \multirow{2}{*}{$\begin{array}{l}\text { Maximum } R_{\text {sum }}^{\mathrm{STRS}} \text {, } \\
\text { for given } \iota\end{array}$} & $\iota=1$ & & & $r_{[F E]}\left(\lambda^{\star}\right),(27),(33)$ \\
\hline & $\iota=2$ & \multicolumn{2}{|l|}{$r_{2 ; b}(1}$, & $r_{2: b}(0,0),(22)$ \\
\hline \multicolumn{2}{|c|}{ Overall maximum $R_{\text {sum }}^{\text {STRS }}: \max \left(R_{\text {sum }}^{\text {STRS }}\right)$} & $r_{1 ; b}(0,1),(22)$ & $r_{[F E]}(0),(27)$ & $r_{[F E]}\left(\lambda^{\star}\right),(27),(33)$ \\
\hline \multicolumn{2}{|l|}{$\Rightarrow$ overall best $\left(\iota ; \lambda_{1}, \lambda_{2}\right)$} & $(1 ; 0,1)$ & $\left(1 ; 0,1-\frac{\beta_{1}}{\beta_{2}}\right),(24)$ & $\left(1 ; \lambda^{\star}, 1-\frac{\beta_{1}}{\beta_{2}}\left(1-\lambda^{\star}\right)\right),(24),(33)$ \\
\hline
\end{tabular}

TABLE I: Maximum STRS sum-rate, $\max \left(R_{\text {sum }}^{\mathrm{STRS}}\right)$, and optimal parameter selection for given $\left(\gamma_{2,2}, \gamma_{1,1}, \gamma_{1,2}, \gamma_{2,1}\right)$.

(15) defines a smaller subset of allowed STRS weight factors within the domain (25). Similar to the above it is now possible to find the pair of weight factors within this particular subset that maximizes the (fair) STRS sum-rate. A detailed derivation and closed-form analytical expressions are provided in the Appendix.

\section{GDOF ANALYSIS}

We now show that the proposed scheme achieves the sumGDoF for the 2-user MISO BC under finite precision CSIT [15].

The sum-GDoF is an approximation of the sum-capacity which is accurate to first order. If the channel link qualities are modelled as $\gamma_{j, k}=P^{\alpha_{j, k}}\left|h_{j, k}\right|^{2}$, where $\alpha_{j, k}>0$ denotes the power scaling exponent for the link between $\mathrm{RX} j$ and TX antenna $k$, then the sum-GDoF is the limit for high transmit power $P$ of the ratio of the sum-capacity $C_{\text {sum }}(P)$ to the reference capacity of a baseline AWGN channel with the same transmit power $P$ and noise variance:

$$
\mathrm{GDoF}_{\text {sum }}=\lim _{P \rightarrow \infty} \frac{C_{\text {sum }}(P)}{\log _{2} P} .
$$

In [15], the sum-GDoF for the 2-user MISO BC with finite precision CSIT was derived as:

$$
\begin{aligned}
\operatorname{GDoF}_{\text {sum }}=\min ( & \max \left(\alpha_{1,1}, \alpha_{1,2}\right)+\max \left(0, d_{1}, d_{2}\right), \\
& \left.\max \left(\alpha_{2,1}, \alpha_{2,2}\right)+\max \left(0,-d_{1},-d_{2}\right)\right)
\end{aligned}
$$

where

$$
\begin{aligned}
& d_{1}=\alpha_{2,1}-\alpha_{1,1}, \\
& d_{2}=\alpha_{2,2}-\alpha_{1,2} .
\end{aligned}
$$

In order to perform an asymptotic sum-GDoF analysis of STRS, we revisit the results of Section VI and Table I, where we first substitute $\gamma_{j, k}=P^{\alpha_{j, k}}\left|h_{j, k}\right|^{2}$ with $\alpha_{j, k}>0$ and then let $P$ go to infinity. Taking into account (I1), it appears that, when doing so, the upper and lower boundaries in (31) converge to each other for large $P$. The same holds for the upper and lower boundaries in (29) if $\alpha_{1,2} \leq \alpha_{1,1}$. Therefore, as far as the asymptotic sum-GDoF analysis is concerned, we can limit our attention to the following cases.

- Condition (30) holds. It can be verified that, for $P$ going to infinity, (30) implies

$$
\alpha_{1,1}<\alpha_{2,1} \leq \alpha_{1,1}+\alpha_{2,2}-\alpha_{1,2} .
$$

Taking (37) into account, the sum-GDoF (36) can be rewritten as

$$
\mathrm{GDoF}_{\text {sum }}=\max \left(\alpha_{2,2}, \alpha_{2,1}\right) .
$$

On the other hand, as reported in Table I, the maximum STRS sum-rate in this case is $r_{[E F]}(0)$ from (27). Using (27) and (21), it is easily verified that

$$
\begin{aligned}
& \lim _{P \rightarrow \infty} \frac{\max \left(R_{\text {sum }}^{\mathrm{STRS}}\right)}{\log _{2} P}=\lim _{P \rightarrow \infty} \frac{r_{[E F]}(0)}{\log _{2} P} \\
& =\lim _{P \rightarrow \infty} \frac{\log _{2}\left(1+\left|h_{2,2}\right|^{2} P^{\alpha_{2,2}}+\left|h_{2,1}\right|^{2} P^{\alpha_{2,1}}\right)}{\log _{2} P} \\
& =\max \left(\alpha_{2,2}, \alpha_{2,1}\right) .
\end{aligned}
$$

Since (38) and (39) yield the same result, we conclude that STRS is indeed optimum in the sense that it achieves the maximum sum-GDoF, if (30) holds.

- Condition (29) holds and $\alpha_{1,2}>\gamma_{1,1}$. Taking into account (I1), it can be shown that, for $P$ going to infinity, (29) with $\alpha_{1,2}>\gamma_{1,1}$ implies

$$
\alpha_{2,1} \leq \alpha_{1,1}<\alpha_{1,2} \leq \alpha_{2,2} .
$$

If (40) holds, the sum-GDoF (36) again reduces to (38). This immediately proves the sum-GDoF optimality of STRS if (29) holds, as the maximum STRS sum-rate is the same for (29) and (30).

- Condition (28) holds. For $P$ going to infinity, (28) in combination with (I1)-(I2) implies

$$
\left\{\begin{array}{ll}
\alpha_{2,1} \leq \alpha_{1,1}<\alpha_{1,2} \leq \alpha_{2,2} & , \text { if } \gamma_{1,2}>\gamma_{1,1} \\
\max \left(\alpha_{1,2}, \alpha_{2,1}\right) \leq \alpha_{1,1}<\alpha_{2,2} & , \text { if } \gamma_{1,2} \leq \gamma_{1,1}
\end{array} .\right.
$$

From Table I, we recall that, if (28) holds, the maximum STRS sum-rate is $r_{[E F]}\left(\lambda^{\star}\right)$ with $r_{[E F]}(\cdot)$ from (27) (using (21)) and $\lambda^{\star}$ as in (33). Using this maximum sum-rate expression, it can be shown that $\lim _{P \rightarrow \infty} \frac{\max \left(R_{s u m}^{\mathrm{STRS}}\right)}{\log _{2} P}$ indeed converges to the sum-GDoF (36) if either line of (41) holds. We obtain

$$
\lim _{P \rightarrow \infty} \frac{\max \left(R_{\text {sum }}^{\mathrm{STRS}}\right)}{\log _{2} P}=\mathrm{GDoF}_{\text {sum }}
$$

with

$$
\mathrm{GDoF}_{\text {sum }}= \begin{cases}\alpha_{2,2}, & \alpha_{1,2}>\alpha_{1,1} \geq \alpha_{2,1} \\ \alpha_{1,1}+\alpha_{2,2}-\phi, & \alpha_{1,1} \geq \phi \\ \phi=\max \left(\alpha_{1,2}, \alpha_{2,1}\right) . & \end{cases}
$$

All in all, we conclude that STRS achieves the sum-GDoF of the 2-user MISO BC under finite precision CSIT.

\section{NUMERICAL RESULTS}

This section presents and discusses some numerical results on the STRS sum-rate and achievable rate-region. In order to get some initial insight, first we address the symmetric case, 
with $\gamma_{1,1}=\gamma_{2,2} \triangleq$ snr, $\gamma_{1,2}=\gamma_{2,1} \triangleq$ inr and snr $\geq$ inr. It follows from Section VI that, in this case, the maximum achievable STRS sum-rate is given by

$$
\max \left(R_{\text {sum }}^{\mathrm{STRS}}\right)=\log _{2}\left(\frac{(1+\mathrm{snr}+\mathrm{inr})(\mathrm{snr}+\mathrm{inr})^{2}}{4 \cdot \mathrm{snr} \cdot \mathrm{inr}}\right)
$$

with

$$
\lambda_{1}=\lambda_{2}=\min \left\{\frac{\mathrm{snr}-\mathrm{inr}}{\mathrm{inr}(\mathrm{snr}+\mathrm{inr})}, 1\right\} .
$$

From (44) it follows that, in the symmetric case, the optimum private rates $R_{1, p}$ and $R_{2, p}$ from (12)-(13) are equal. As a result, the maximum STRS sum-rate can always be achieved with identical rates for both users (i.e., $R_{1}=R_{2}$, see (10)(11)). The sum-rate from (43) is necessarily not worse than that for the HK scheme, when both TX antennas cannot cooperate. In Fig. 3, the ratio of the maximum sum-rate with respect to the reference capacity of an AWGN channel, $C(\mathrm{snr})=\log _{2}(1+\mathrm{snr})$, is depicted together with the optimal weighting factor $\lambda_{1}=\lambda_{2}=\lambda$ for both STRS and HK, versus the ratio $\log \mathrm{inr} / \log \mathrm{snr}$, for $\mathrm{snr}=15 \mathrm{~dB}$. The expression of the corresponding HK lower bound for the sum-rate in the symmetric case is found in [3, Ex. 6.16]:

$$
\begin{aligned}
\max \left(R_{\text {sum }}^{\mathrm{HK}}\right)= & \max _{\lambda \in[0,1]} \min \left\{2 \log _{2}\left(1+\frac{\mathrm{snr}}{1+\lambda \cdot \mathrm{inr}}\right),\right. \\
& 2 \log _{2}\left(1+\frac{\lambda \cdot \mathrm{snr}+(1-\lambda) \cdot \mathrm{inr}}{1+\lambda \cdot \mathrm{inr}}\right), \\
& \log _{2}\left(1+\frac{\mathrm{snr}+(1-\lambda) \mathrm{inr}}{1+\lambda \cdot \mathrm{inr}}\right)+ \\
& \left.\log _{2}\left(1+\frac{\lambda \cdot \mathrm{snr}}{1+\lambda \cdot \mathrm{inr}}\right)\right\} .
\end{aligned}
$$

The gain of STRS with respect to HK, which is relevant for intermediate interference regimes, comes from the cooperation when encoding the two messages $m_{1}$ and $m_{2}$ and synthesizing the transmit symbols $x_{1}[k]$ and $x_{2}[k]$ in Fig. 1. Note that this gain is a consequence of that anticipated by the GDoF framework. Following [15], the symmetric setting can be asymptotically defined with one parameter, so that $\alpha_{1,1}=\alpha_{2,2}=1, \alpha_{1,2}=\alpha_{2,1}=\alpha_{\text {sym }}$ in (4). With this, the sum-GDoF of the finite precision CSIT BC is given by $2-\alpha_{\text {sym }}$, whereas that of the $\mathrm{IC}^{4}$ is given by $\min \left\{2-\alpha_{\text {sym }}, 2 \max \left\{\alpha_{\text {sym }}, 1-\alpha_{\text {sym }}\right\}\right\}$. The sum-GDoF is also plotted in Fig. 3a for reference purposes.

Next, we show the achievable rate region of STRS using for comparison the following baseline metrics: (i) DPC with perantenna power constraints (DPC-pac) [4]; (ii) ENH [15]; (iii) SC [21]; (iv) frequency-division multiplexing (FDM); and (v) time-sharing (TS). The complexity of each scheme, together with the amount of required CSIT for operation, are collected in Table II. As a reference, the single-user MISO capacity under per-antenna power constraint and full CSIT (MISO-pac) [22] is also depicted.

The channel coefficients have been generated with random phases following a uniform distribution, with the magnitudes

\footnotetext{
${ }^{4}$ In the case of the IC, there is no GDoF loss of finite precision CSIT relative to full CSIT [15].
}

as labeled above each sub-plot in Fig. 4. Even though the pairing of users at system level is not discussed in this work, different channel magnitudes are simulated to illustrate the potential under different circumstances in terms of high/low interference or strong/mild user unbalance. In the case of $\mathrm{ENH}$, the average weighted sum-rate is used to plot the achievable rate region. This approach is followed in [5] to maximize the ergodic rate. In our case we are interested in the instantaneous rates, which cannot be predicted by ENH under our CSIT model, due to the phase uncertainty. For illustration purposes, a genie-aided design is considered, since phases are assumed to be known to optimize the ENH weights at each channel realization. This could be overcome to some extent with a worst-case approach like that in [20], at the cost of decreasing the shown rates. Even this ENH genie-aided approach performs worse than the proposed STRS, which performs independently of the channel phase.

The gain of STRS with respect to SC is due to the knowledge of the magnitude of the individual links, which allows to optimize the amount of information coming out of each antenna to a given terminal. The relative powers $\lambda_{1}$ and $\lambda_{2}$ which maximize the STRS achievable sum-rate according to the optimization in Section VI are reported in the figure caption. In all cases, the direct links have better SNRs than the leakage links, and the direct link SNRs are significantly larger than $0 \mathrm{~dB}$. In correspondence to the intermediate discussion in Section VI, the optimal weight factor is more or less the same for both TX antennas. Further, as expected, more power should be allocated to the common message when the leakage link SNRs increase (interference becomes more relevant). Depending on the specific setting, the gap of the reported schemes till the channel capacity with full CSIT can be significant, and it remains an open problem to determine whether the rate region achieved by STRS can be further improved with some alternative encoding scheme.

Some additional examples are presented in Fig. 5 to illustrate the trade-off between fairness and sum-rate; HanKobayashi (HK) and single-user (SU) performance are also plotted. The values of $\left(\lambda_{1}, \lambda_{2}\right)$ that yield the maximum STRS sum-rate, $\max \left(R_{\text {sum }}^{\mathrm{STRS}}\right)$ (Section VI) and the maximum STRS sum-rate when fairness as $R_{1}=R_{2}$ is imposed, $\max \left(R_{\text {sum } ; R_{1}=R_{2}}^{\mathrm{STRS}}\right)$, (Appendix), respectively, are reported in the figure caption. It can be seen that the optimum $\left(\lambda_{1}, \lambda_{2}\right)$ values are lower when fairness is imposed: to ensure fairness more power needs to be allocated to the common message. Note that in all four sub-plots of Fig. 5 the CSIT MISO BC is significantly unbalanced. As a result, equal rates for both users are not compatible with the maximum sum-rate solution provided by STRS, despite the fact that the maximum sum-rate is typically achieved for multiple $\left(R_{1}, R_{2}\right)$ pairs.

\section{CONClusions}

The two-user vector broadcast channel has been addressed under the absence of phase information at a two-antenna transmitter (TX). A rate splitting approach between private and common messages, named STRS, and properly optimized, has been used to exploit the knowledge of the magnitude of the 


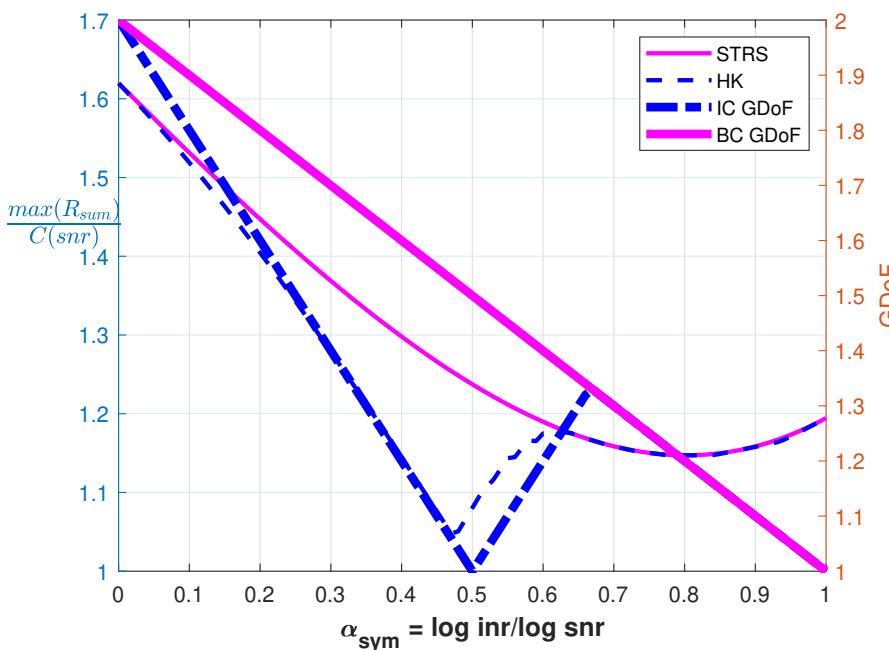

(a)

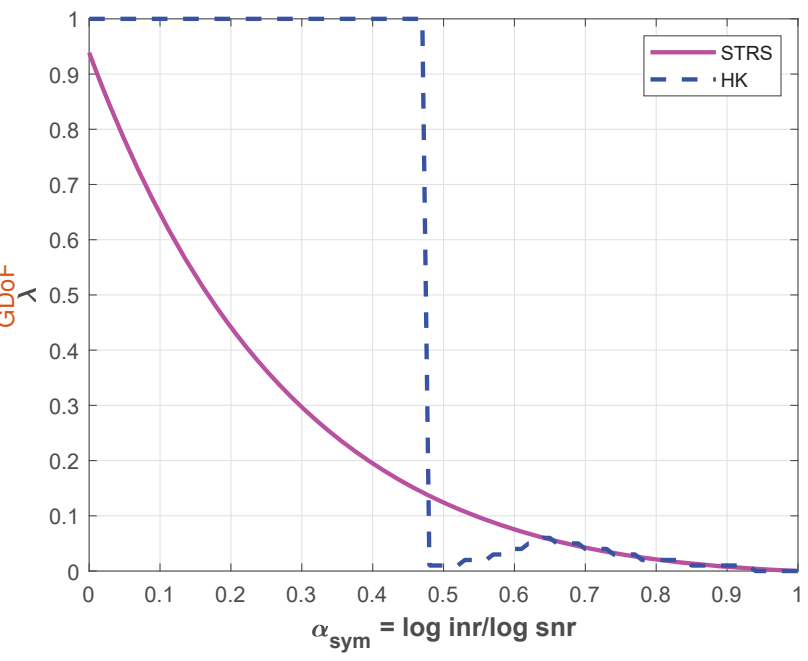

(b)

Fig. 3: Comparison of STRS and HK in the symmetric case, snr $=15 \mathrm{~dB}$. (a) Sum-rate. (b) Weighting factor.

TABLE II: Requirements of different multiuser transmission schemes.

\begin{tabular}{|c|c|c||c|c|c|}
\hline Scheme & Amount of CSIT & Complexity & Scheme & Amount of CSIT & Complexity \\
\hline ENH & Finite Precision & Medium & SC & Total channel magnitude & Medium \\
\hline DPC-pac & Full & High & FDM & no CSIT & Low \\
\hline STRS & Channel coefficients magnitude & Medium & TS & no CSIT & Low \\
\hline
\end{tabular}

individual paths, and compared with previously known results in the literature which share only some of the constraints of the problem under study. Rate allocation fairness has been also explored, by exploiting the seamless rate allocation of STRS, so that the sum-rate can be maximized while still providing the same rate to both users unless their respective channels are significantly unbalanced. The new scheme has been proved to achieve the sum-GDoF, and yields achievable rates which are independent of the channel phase, which makes it a good match for practical link adaptation. Finally, it is an open problem to design new schemes able to enlarge the STRS rate region in the absence of channel phase information.

\section{ACKnOWledgments}

This work has been partially supported by European Space Agency funded activity SatNEx IV CoO2-Part 1 WI 3, "NonOrthogonal Superposition Techniques for Multi-Beam Satellite Networks". The views of the authors of this paper do not reflect the views of ESA. Also funded by the Agencia Estatal de Investigación (Spain) and the European Regional Development Fund (ERDF) through the project RODIN (PID2019105717RB-C21).

\section{APPENDIX}

To complement Section VI, we derive analytical expressions for the values $\iota, \lambda_{1}$ and $\lambda_{2}$, for which the STRS sum-rate $R_{\text {sum }}^{\mathrm{STRS}}$ from (20) achieves a maximum when equal user rates fairness is imposed as an additional constraint (see (10)-(15)). We will denote this maximum as $\max \left(R_{\text {sum } ; R_{1}=R_{2}}^{\mathrm{STRS}}\right)$.

\section{A. Relaxed problem definition}

To simplify the derivation, we first replace the fairness constraint in (15) with a less restricted set of operation points:

$\mathbb{A} \triangleq\left\{\left(\lambda_{1}, \lambda_{2}\right) \in[0,1]^{2}: \lambda_{1} \geq \mathcal{A}_{1}\left(\lambda_{2}\right)\right.$ and $\left.\lambda_{2} \geq \mathcal{A}_{2}\left(\lambda_{1}\right)\right\}$.

Here, $\mathcal{A}_{1}(x)$ and $\mathcal{A}_{2}(x)$ are auxiliary functions, defined as

$$
\mathcal{A}_{i}(x) \triangleq \frac{Q_{i}}{\gamma_{3-i, i}\left(\gamma_{i, i}+(1-x) \gamma_{i, 3-i}\right)},
$$

with

$Q_{i}=x^{2} \gamma_{i, 3-i} \gamma_{3-i, 3-i}+x\left(\gamma_{3-i, 3-i}+\gamma_{i, 3-i}\right)-\left(\gamma_{i, i}+\gamma_{i, 3-i}\right)$.

A relaxed optimization problem results, which boils down to finding the maximum of $R_{\text {sum }}^{\mathrm{STRS}}$ over $\iota \in\{1,2\}$ and over the set $\mathbb{A}$ from (45). In general, (45) is a necessary but not sufficient condition for (15); an additional explanation and the rationale behind this criterion is provided in the next subsection. Obviously, if the solution to the relaxed optimization problem using the constraint (45) turns out to obey (15), then it is also the solution to the original constrained optimization problem. As it turns out, this will be exactly the case.

\section{B. Rationale behind fairness constraint relaxation}

Without loss of generality, we select $i \in\{1,2\}$, so that

$$
\beta_{3-i}\left(1-\lambda_{3-i}\right) \geq \beta_{i}\left(1-\lambda_{i}\right)
$$




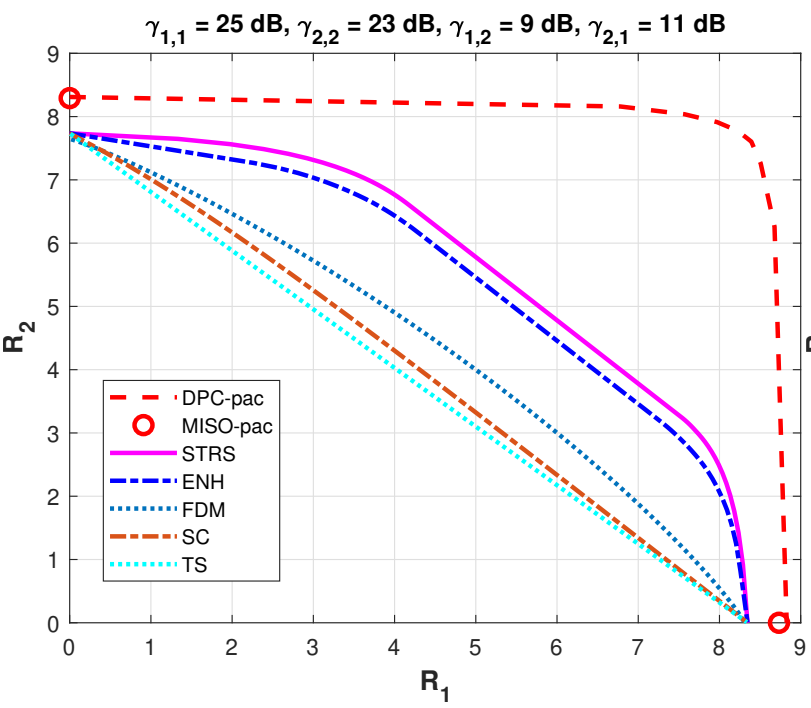

(a)

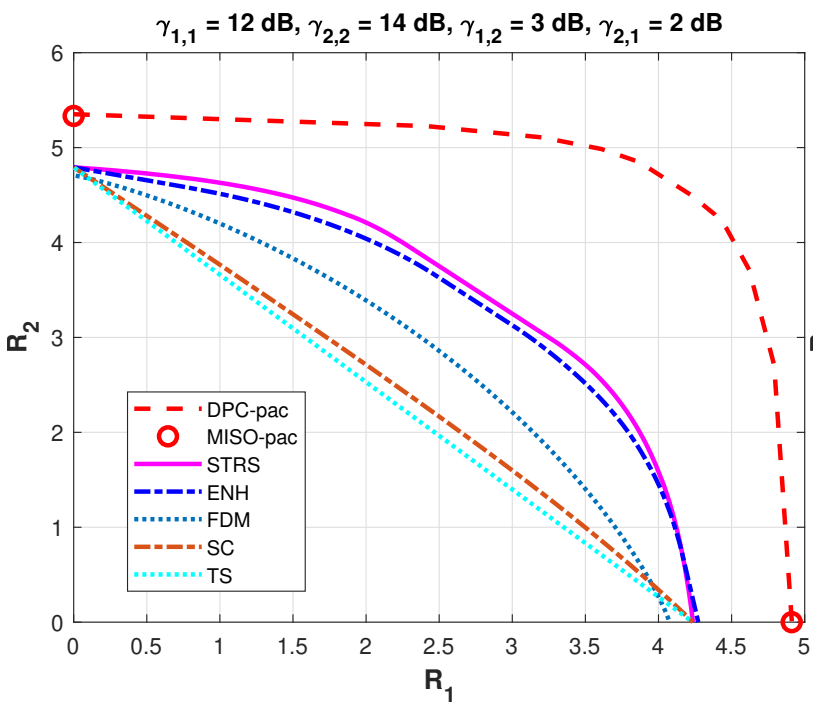

(c)

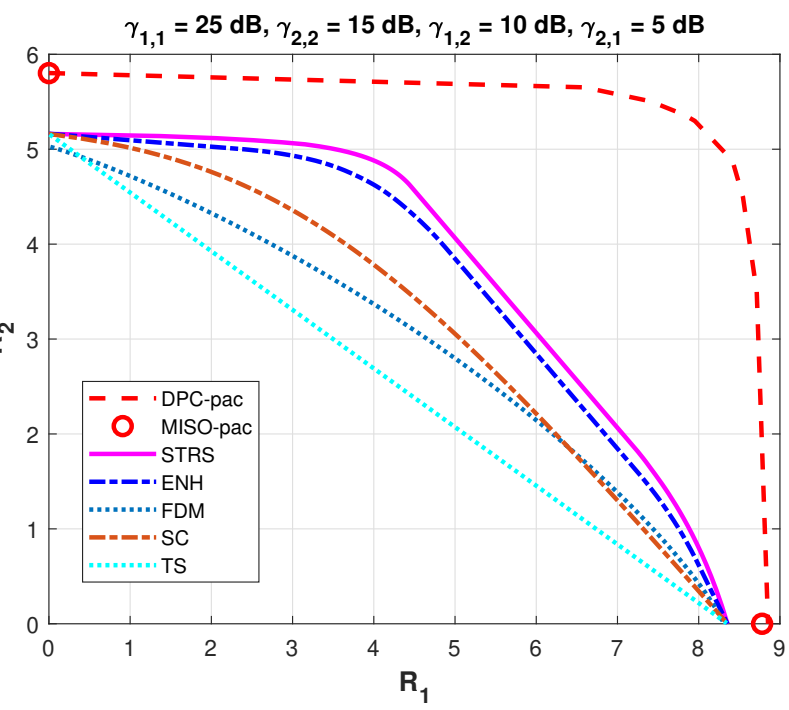

(b)

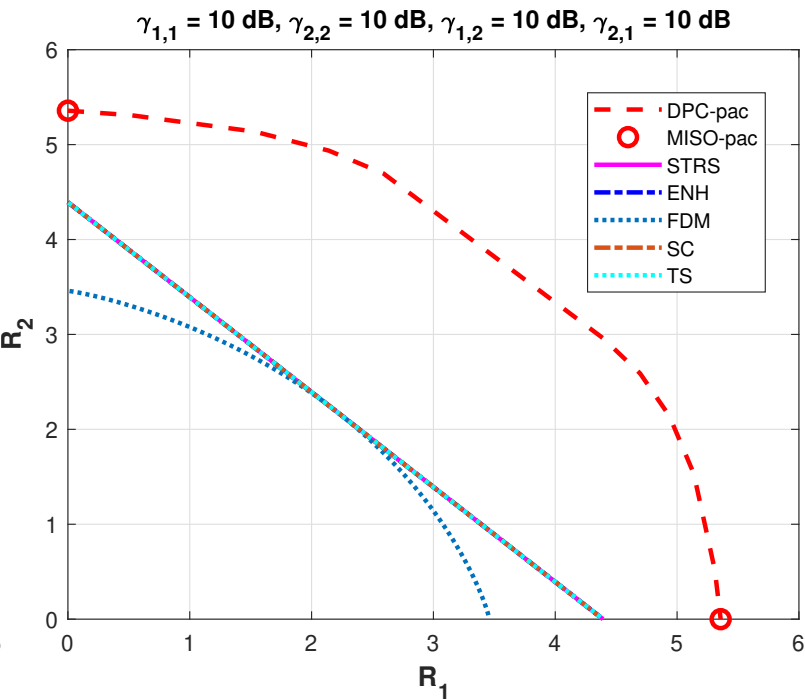

(d)

Fig. 4: Achievable rate regions of STRS and different baseline schemes. The ENH curves are included for completeness: they correspond to an upper bound for the ergodic rates, since the channel phase is assumed to be known for the optimization of the messages' weights in ENH at each channel realization. $R_{1}$ and $R_{2}$ are given in bps/Hz. In (d), STRS, ENH, SC and TS performance is identical. Maximum STRS sum-rate is achieved for $\iota=1$, and the weights maximizing the sum-rate of STRS are: (a) $\lambda_{1}=0.093, \lambda_{2}=0.091$; (b) $\lambda_{1}=0.14, \lambda_{2}=0.12$; (c) $\lambda_{1}=0.46, \lambda_{2}=0.47$; (d) $\lambda_{1}=0, \lambda_{2}=1$. In (d), STRS yields the same maximum sum-rate for $\iota=1, \lambda_{1}=0$ and any $\lambda_{2} \in[0,1]$.

in the following. After substituting (12)-(13), the fairness constraint (15) can be rewritten as

$$
\left\{\begin{array}{l}
R_{c} \geq \log _{2}\left(\frac{\left(1+\lambda_{i} \gamma_{i, i}+\lambda_{3-i} \gamma_{i, 3-i}\right)\left(1+\lambda_{i} \gamma_{3-i, i}\right)}{\left(1+\lambda_{i} \gamma_{3}-i, i+\lambda_{3-i} \gamma_{3-i, 3-i}\right)\left(1+\lambda_{3-i} \gamma_{i, 3-i}\right)}\right) \\
R_{c} \geq \log _{2}\left(\frac{\left(1+\lambda_{i} \gamma_{3-i, i}+\lambda_{3-i} \gamma_{3-i, 3-i}\right)\left(1+\lambda_{3-i} \gamma_{i, 3-i}\right)}{\left(1+\lambda_{i} \gamma_{i, i}+\lambda_{3-i} \gamma_{i, 3-i}\right)\left(1+\lambda_{i} \gamma_{3-i, i}\right)}\right)
\end{array} .\right.
$$

From (14) and (47), we obtain

$$
\begin{gathered}
R_{c}=\log _{2}\left(\frac{1+\gamma_{i, i}+\gamma_{i, 3-i}}{1+\lambda_{i} \gamma_{i, i}+\lambda_{3-i} \gamma_{i, 3-i}}\right), \\
R_{c} \leq \log _{2}\left(\frac{1+\gamma_{3-i, 3-i}+\gamma_{3-i, i}}{1+\lambda_{3-i} \gamma_{3-i, 3-i}+\lambda_{i} \gamma_{3-i, i}}\right) .
\end{gathered}
$$

Using (48)-(50) it then follows that (15) implies

$$
\left\{\begin{array}{l}
\frac{1+\gamma_{3-i, 3-i}+\gamma_{3-i, i}}{1+\lambda_{3-i} \gamma_{3-i, 3-i}+\lambda_{i} \gamma_{3-i, i}} \geq \Phi \\
\frac{1+\gamma_{i, i}+\gamma_{i, 3-i}}{1+\lambda_{i} \gamma_{i, i}+\lambda_{3-i} \gamma_{i, 3-i}} \geq \frac{1}{\Phi} \\
\Phi=\frac{\left(1+\lambda_{i} \gamma_{i, i}+\lambda_{3-i} \gamma_{i, 3-i}\right)\left(1+\lambda_{i} \gamma_{3-i, i}\right)}{\left(1+\lambda_{i} \gamma_{3-i, i}+\lambda_{3-i} \gamma_{3-i, 3-i}\right)\left(1+\lambda_{3-i} \gamma_{i, 3-i}\right)}
\end{array}\right.
$$

The first line in (51) follows from (50) and the first line in (48); the second line in (51) follows from (49) and the second line of (48). By means of straightforward operations, it is easily 


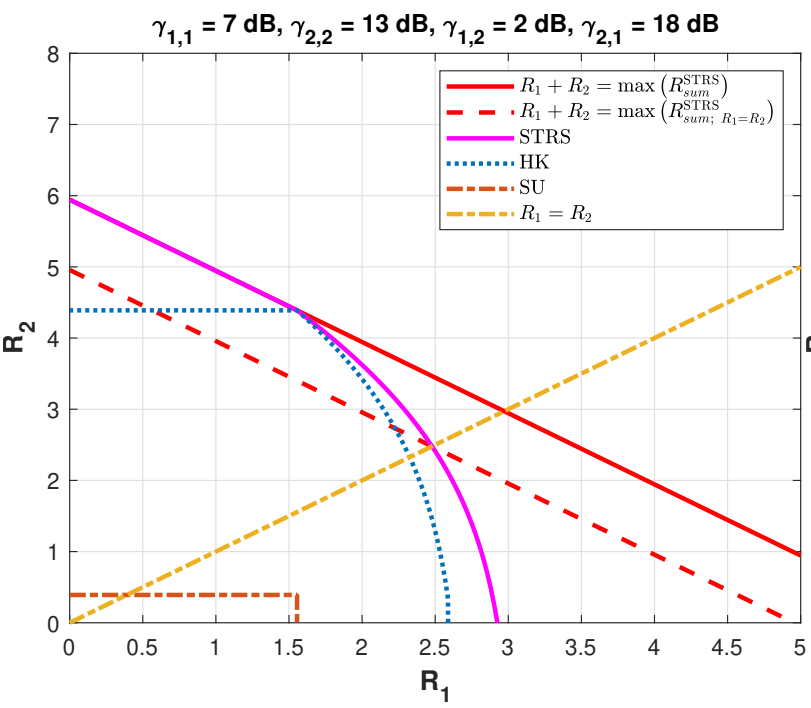

(a)

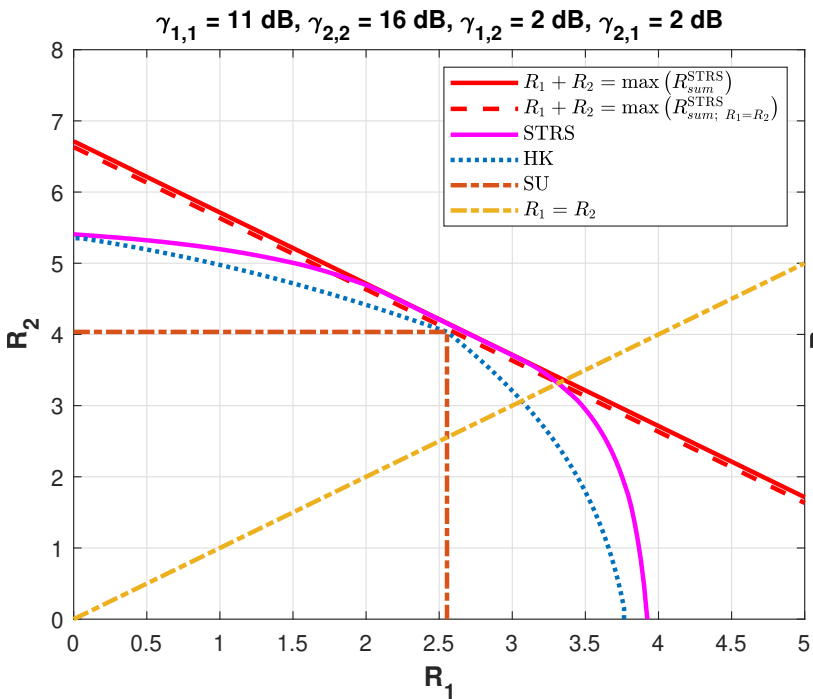

(c)

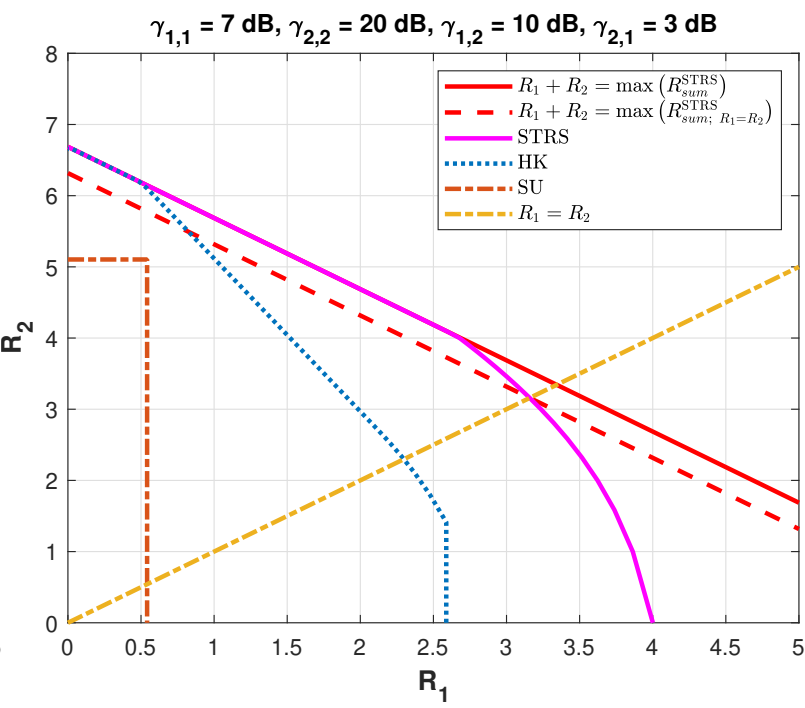

(b)

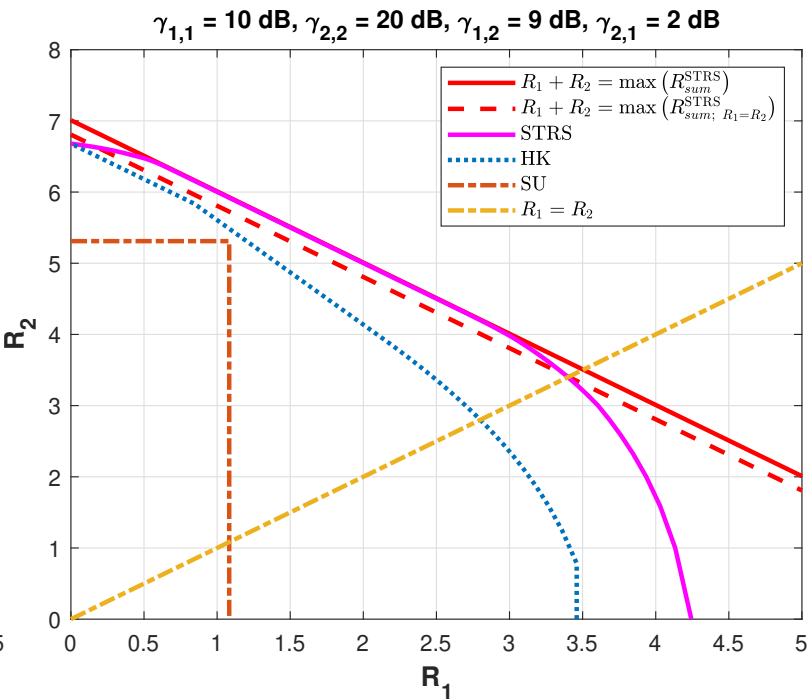

(d)

Fig. 5: Achievable rate regions of STRS, Han-Kobayashi (HK), and single-user (SU) decoding, with respect to the maximum STRS sum-rates, $\max \left(R_{\text {sum }}^{\mathrm{STRS}}\right)$ and $\max \left(R_{\text {sum } ; R_{1}=R_{2}}^{\mathrm{STRS}}\right)$. The weights corresponding to $\max \left(R_{\text {sum }}^{\mathrm{STRS}}\right)$ are: (a) $\lambda_{1}=0, \lambda_{2}=1$; (b) $\lambda_{1}=0, \lambda_{2}=0.15$; (c) $\lambda_{1}=0.52, \lambda_{2}=0.54$; (d) $\lambda_{1}=0.17, \lambda_{2}=0.23$. The weights corresponding to $\max \left(R_{\text {sum }}^{\mathrm{STRS}} R_{1}=R_{2}\right)$ are: (a) $\lambda_{1}=0, \lambda_{2}=0.23$; (b) $\lambda_{1}=0, \lambda_{2}=0.08$; (c) $\lambda_{1}=0.29, \lambda_{2}=0.33$; (d) $\lambda_{1}=0.02, \lambda_{2}=0.10$.

shown that (51) is equivalent to

$$
\left\{\begin{array}{l}
\lambda_{3-i} \geq \mathcal{A}_{3-i}\left(\lambda_{i}\right) \\
\lambda_{i} \geq \mathcal{A}_{i}\left(\lambda_{3-i}\right) .
\end{array}\right.
$$

Although (15) implies (52), the converse does not hold; in general, (52) does not imply (15). This is a consequence of the inequality in (50) that is used to arrive at (51). As a result, (52) (or, equivalently, (45)) is less stringent than the original fairness constraint (15).

\section{Properties of $\mathbb{A}$}

The set $\mathbb{A}$ from (45) demarcates an area in Fig. 2 of Section VI that is bounded by the lines $\lambda_{1}=0, \lambda_{1}=1$, $\lambda_{2}=0, \lambda_{2}=1$ and by the $\operatorname{arcs} a_{i}$, with $i=1,2$ and $a_{i} \triangleq\left\{\left(\lambda_{1}, \lambda_{2}\right): \lambda_{i}=\mathcal{A}_{i}\left(\lambda_{3-i}\right), \lambda_{3-i} \in[0,1]\right\}$. See Fig. 6, where the area $\mathbb{A}$ is shaded and the arcs $a_{1}$ and $a_{2}$ are indicated. From (45)-(46), it can be verified that, for any set of link SNR values $\left(\gamma_{1,1}, \gamma_{1,2}, \gamma_{2,1}, \gamma_{2,2}\right)$,

(P5) The area $\mathbb{A}$ contains the origin.

For all $\lambda_{3-i} \in[0,1], \mathcal{A}_{i}\left(\lambda_{3-i}\right)$ from (46), is a monotonically increasing function of $\lambda_{3-i}$. Hence,

(P6) The area $\mathbb{A}$ collects all the points in the power splitting domain that are to the right of $a_{1}$ and to the left of $a_{2}$, simultaneously.

It can further be verified from (21)-(22) and (46) that

(P7) $\quad r_{1 ; b}\left(\mathcal{A}_{1}\left(\lambda_{2}\right), \lambda_{2}\right)$ and $r_{2 ; a}\left(\mathcal{A}_{1}\left(\lambda_{2}\right), \lambda_{2}\right)$ are decreasing functions of $\lambda_{2}$, for $\lambda_{2} \geq 0$. 
(P8) $\quad r_{1 ; a}\left(\lambda_{1}, \mathcal{A}_{2}\left(\lambda_{1}\right)\right)$ and $r_{2 ; b}\left(\lambda_{1}, \mathcal{A}_{2}\left(\lambda_{1}\right)\right)$ are decreasing functions of $\lambda_{1}$, for $\lambda_{1} \geq 0$.

These properties are also illustrated in Fig. 6, where arrows indicate the direction in which $R_{\text {sum }}^{\mathrm{STRS}}$ increases when moving along a given trajectory. As $a_{1}$ and $a_{2}$ are increasing curves, (P7) implies that, for $\iota=1(\iota=2), R_{\text {sum }}^{\mathrm{STRS}}$ increases when moving in the direction of the origin along any part of the arc $a_{1}$ below (above) the line $F E$. Similarly, (P8) implies that, for $\iota=1(\iota=2), R_{\text {sum }}^{\mathrm{STRS}}$ increases when moving in the direction of the origin along any part of the arc $a_{2}$ above (below) the line $F E$. For simplicity, the direction of increasing $R_{\text {sum }}^{\mathrm{STRS}}$ along the $\lambda_{1}$-axis is not indicated in Fig. 6, but the behaviour of $R_{\text {sum }}^{\mathrm{STRS}}$ along this axis is as indicated in Fig. 2.

\section{Case $\beta_{2} \geq 0 \geq \beta_{1}$}

If $\beta_{2} \geq 0 \geq \beta_{1}$, the situation is the one depicted by the plots in the first column of Fig. 6, but with the $\lambda_{2}$-coordinate value of the point $F$ larger than 1 . Noting that $\beta_{2} \geq 0 \geq \beta_{1}$ implies $\gamma_{1,1} \leq \gamma_{2,1}$, we easily obtain that, in this case, the maximum achievable $R_{\text {sum }}^{\mathrm{STRS}}$ over $\mathbb{A}$ is

$$
R_{\text {sum }}^{\mathrm{STRS}}=\max \left(r_{1 ; b}\left(0, \min \left(\tilde{\lambda}_{2}, 1\right)\right), r_{2 ; b}\left(\min \left(\tilde{\lambda}_{1}, 1\right), 0\right)\right),
$$

with, for $i \in\{1,2\}, \tilde{\lambda}_{3-i}$ the $\lambda_{3-i}$-coordinate of the intersection of the arc $a_{i}$ and the $\lambda_{3-i}$-axis:

$$
\tilde{\lambda}_{3-i}=\tilde{\lambda}\left(\gamma_{i, i}, \gamma_{i, 3-i}, \gamma_{3-i, i}, \gamma_{3-i, 3-i}\right),
$$

with

$$
\tilde{\lambda}(p, q, r, s)=\frac{-q-s+\sqrt{(q+s)^{2}+4 q s(p+q)}}{2 q s} .
$$

Expression (53) follows from (P1)-(P8) (with (P1)-(P4) specified in Section VI). It is easily verified that (53) satisfies (15). Depending on the outcome of the maximum in (53), the optimum parameter settings are $\left(\iota ; \lambda_{1}, \lambda_{2}\right)=\left(1 ; 0, \min \left(\tilde{\lambda}_{2}, 1\right)\right)$ or $\left(\iota ; \lambda_{1}, \lambda_{2}\right)=\left(2 ; \min \left(\tilde{\lambda}_{1}, 1\right), 0\right)$.

\section{E. Case $\beta_{2} \geq \beta_{1}>0$}

Depending on the value of the discriminant $D$ of the quadratic form that results from setting $\lambda_{1}=$ $\mathcal{A}_{1}\left(1-\frac{\beta_{1}}{\beta_{2}}\left(1-\lambda_{1}\right)\right)$, the arc $a_{1}$ can have 0 to 2 points in common with the line $F E$. The two potential intersections of $a_{1}$ with $F E$ are denoted $\dot{\lambda}_{1}$ and $\ddot{\lambda}_{1}$ (with $\dot{\lambda}_{1} \leq \ddot{\lambda}_{1}$ ). Further, the arc $a_{2}$ always intersects once with the ray $\overrightarrow{F E}$. The $\lambda_{1}$ coordinate of the intersection point of $a_{2}$ and $\overrightarrow{F E}$ is denoted by $\bar{\lambda}_{1}$. The $\lambda_{1}$-coordinate values $\bar{\lambda}_{1}, \dot{\lambda}_{1}$ and $\ddot{\lambda}_{1}$ are indicated in Fig. 6. Closed-form expressions for $\left(\bar{\lambda}_{1}, \dot{\lambda}_{1}, \ddot{\lambda}_{1}, D\right)$ are provided in next subsection. It can be shown that

(P9) If $D \leq 0, a_{1}$ does not intersect with $F E$. For $D<0$, the entire line segment $[F E]$ is located to the left of $a_{1}$. Such a situation is shown in the first column of Fig. 6. In the special case where $D$ equals zero, $F E$ is a tangent to $a_{1}$.

(P10) If $D>0$, there are 2 intersection points at $\lambda_{1}=\dot{\lambda}_{1}$ and $\lambda_{1}=\ddot{\lambda}_{1}$, with $\dot{\lambda}_{1}$ smaller than $\ddot{\lambda}_{1}$. Only the points on the line segment $[F E]$ with a $\lambda_{1}$-coordinate between $\dot{\lambda}_{1}$ and $\ddot{\lambda}_{1}$ are located to the right of $a_{1}$. This is the situation shown in the second and third column of Fig. 6.

(P11) On the line segment $[F E]$ in Fig. 6, only the points with a $\lambda_{1}$-coordinate smaller than $\bar{\lambda}_{1}$ are located to the left of $a_{2}$.

In addition, a direct consequence of the discussion in Section $\mathrm{VI}$ is the following:

(P12) For $\iota=1$, the value of $R_{\text {sum }}^{\mathrm{STRS}}$ increases along the line segment $[F E]$ when approaching the point with $\lambda_{1}$ coordinate equal to $\lambda_{1}^{\star}=\max \left(0, \lambda^{\star}\right)$, with $\lambda^{\star}$ from (33).

For simplicity, the point on $[F E]$ with $\lambda_{1}$ coordinate $\lambda_{1}^{\star}$ is not labelled and the direction of increasing $R_{\text {sum }}^{\mathrm{STRS}}$ along $[F E]$ is not indicated in Fig. 6. Instead, Fig. 7 illustrates the direction of increasing $R_{\text {sum }}^{\mathrm{STRS}}$ on the line segment $[F E]$ in the plots in the second column of Fig. 6, for the different relative positions of the point with $\lambda_{1}$-coordinate $\lambda_{1}^{\star}$ with respect to the points with $\lambda_{1}$-coordinates $\dot{\lambda}_{1}, \ddot{\lambda}_{1}$ and $\bar{\lambda}_{1}$.

From properties (P5)-(P11), we deduce that $\mathbb{A}$ takes only three types of shapes, depicted in columns 1 to 3 of Fig. 6, respectively. We note that $\bar{\lambda}_{1}$ can become larger than 1 , and that $\dot{\lambda}_{1}$ and $\ddot{\lambda}_{1}$ are not necessarily confined to $[0,1]$. The essential features in Fig. 6 are outlined below.

- The plots in the first column of Fig. 6 correspond to a scenario where $\mathbb{A}$ does not contain any point of $[F E]$. This is the case for $(D \leq 0),\left(D>0, \ddot{\lambda}_{1} \leq 0\right)$ and $\left(D>0, \dot{\lambda}_{1} \geq \bar{\lambda}_{1}\right)$. It can be seen that, for $\iota=1$, the maximum of $R_{\text {sum }}^{\mathrm{STRS}}$ over $\mathbb{A}$ is achieved in $\left(0, \tilde{\lambda}_{2}\right)$, while, for $\iota=2$, the maximum of $R_{\text {sum }}^{\mathrm{STRS}}$ over $\mathbb{A}$ is achieved in $(0,0)$ for $\gamma_{1,1} \geq \gamma_{2,1}$ or in $\left(\tilde{\lambda}_{1}, 0\right)$ for $\gamma_{1,1} \leq \gamma_{2,1}$.

- The plots in the second column of Fig. 6 correspond to a scenario where $D>0$ and $\mathbb{A}$ contains the points on $[F E]$ with a $\lambda_{1}$-coordinate in $\left[\left\lfloor\dot{\lambda}_{1}\right\rceil_{0}^{1},\left[\ddot{\lambda}_{1}\right\rceil_{0}^{1}\right]$, with $\left[\left\lfloor\dot{\lambda}_{1}\right\rceil_{0}^{1},\left\lfloor\ddot{\lambda}_{1}\right\rceil_{0}^{1}\right]$ not empty (i.e., $0<\ddot{\lambda}_{1} \leq \bar{\lambda}_{1}$ ). For $\iota=1$, depending on the relative position of $\lambda_{1}^{\star}$ with respect to $\dot{\lambda}_{1}$ and $\ddot{\lambda}_{1}$ (see Fig. 7), and depending on the sign of $\dot{\lambda}_{1}$, the maximum of $R_{\text {sum }}^{\text {STRS }}$ over $\mathbb{A}$ is achieved in $\left(0, \min \left(\tilde{\lambda}_{2}, 1-\frac{\beta_{1}}{\beta_{2}}\right)\right),\left(\lambda_{1}^{\star}, \lambda_{2}^{\star}\right)$ or $\left(\ddot{\lambda}_{1}, 1-\frac{\beta_{1}}{\beta_{2}}\left(1-\ddot{\lambda}_{1}\right)\right)$. For $\iota=2$, the maximum of $R_{\text {sum }}^{\mathrm{STRS}}$ over $\mathbb{A}$ is again achieved in $(0,0)$ for $\gamma_{1,1} \geq \gamma_{2,1}$ or in $\left(\tilde{\lambda}_{1}, 0\right)$ for $\gamma_{1,1} \leq \gamma_{2,1}$.

- The scenario depicted in the plots in the third column of Fig. 6 is very similar to the scenario depicted in the plots in the second column of Fig. 6, but with $\dot{\lambda}_{1}<\bar{\lambda}_{1} \leq \ddot{\lambda}_{1}$ rather than $0<\ddot{\lambda}_{1} \leq \bar{\lambda}_{1}$. A similar reasoning can be applied.

It can be verified that the coordinates of each of these maxima obey (15). Hence, summarizing the above results yields a maximum achievable fair $\left(R_{1}=R_{2}\right)$ STRS sum-rate

$$
\begin{aligned}
& \max \left(R_{\text {sum } ; R_{1}=R_{2}}^{\mathrm{STRS}}\right) \\
& = \begin{cases}\max \left(r_{1 ; b}\left(\lambda_{1}^{\star \star}, \lambda_{2}^{\star \star}\right), r_{2 ; b}\left(\tilde{\lambda}_{1}, 0\right)\right) & , \gamma_{1,1} \geq \gamma_{2,1} \\
\max \left(r_{1 ; b}\left(\lambda_{1}^{\star \star}, \lambda_{2}^{\star \star}\right), r_{2 ; b}(0,0)\right) & , \gamma_{1,1} \leq \gamma_{2,1}\end{cases}
\end{aligned}
$$



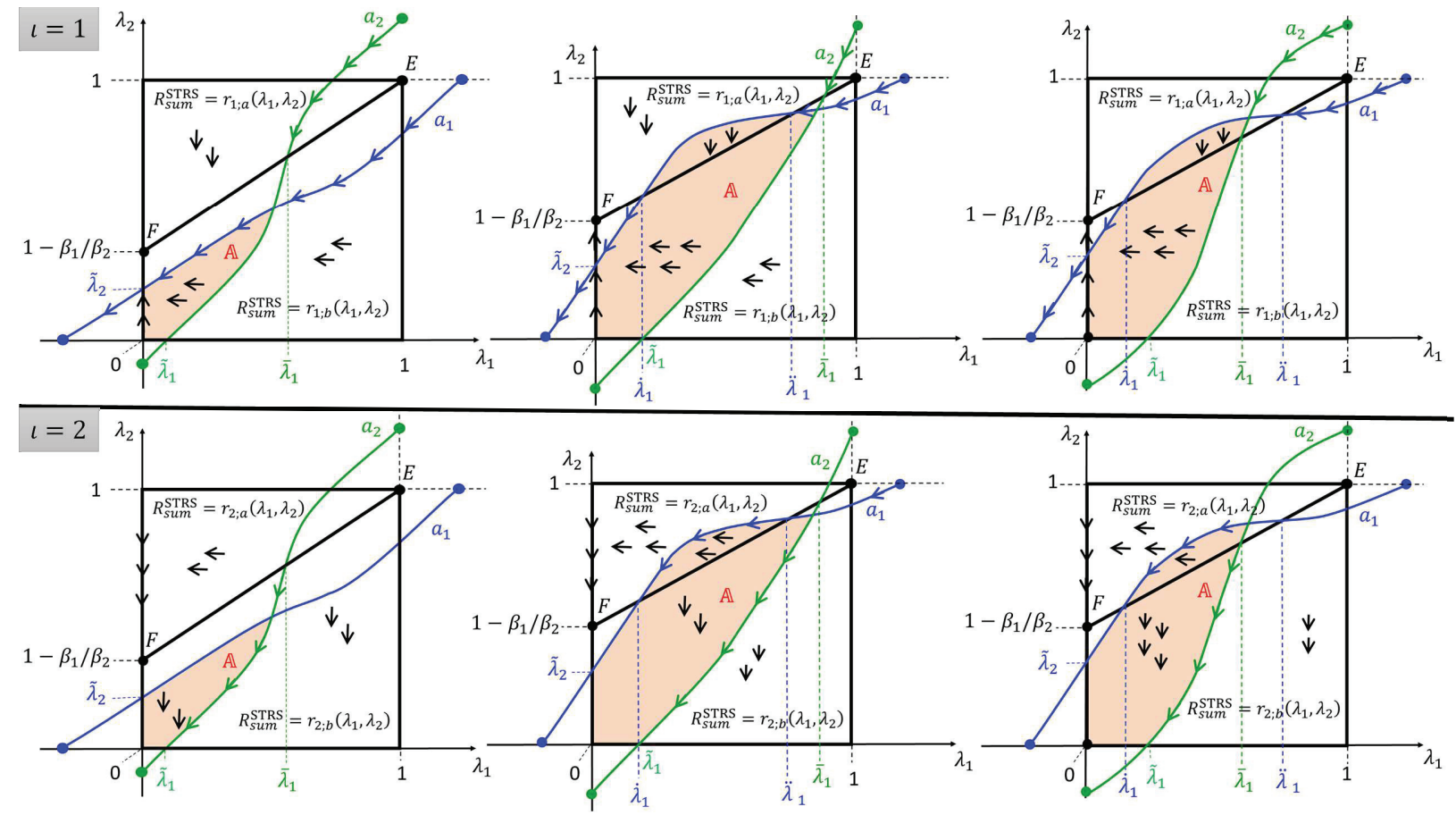

Fig. 6: Search area $\mathbb{A}$, bounding arcs $a_{1}$ and $a_{2}$ and arrows indicating increasing $R_{\text {sum }}^{\text {STRS }}$ for $\beta_{2} \geq \beta_{1}>0$, for $\iota=1$ (top) or $\iota=2$ (bottom). Column 1: $[F E]$ is to the left of $a_{1}$ for $\lambda_{1} \in\left[0, \bar{\lambda}_{1}\right]$. Column 2: $D \geq 0$ and $\max \left(0, \dot{\lambda}_{1}\right)<\ddot{\lambda}_{1} \leq \bar{\lambda}_{1}$. Column 3: $D \geq 0, \dot{\lambda}_{1} \leq \bar{\lambda}_{1} \leq \ddot{\lambda}_{1}$.

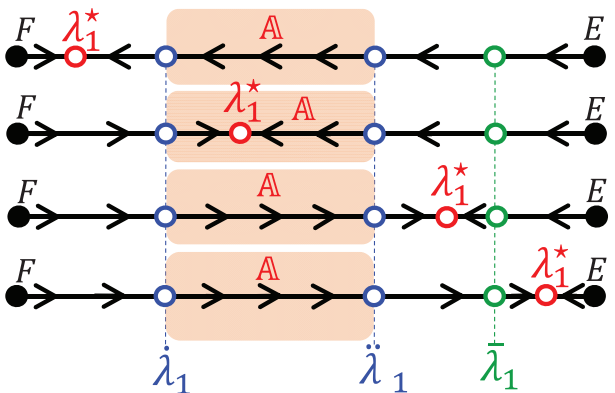

Fig. 7: Line segment $[F E]$ with the position of the points with $\lambda_{1}$-coordinate equal to $\dot{\lambda}_{1}, \ddot{\lambda}_{1}$ and $\bar{\lambda}_{1}$ as in the second column of Fig. 6, for 4 hypotheses on the relative position of the point with $\lambda_{1}=\lambda_{1}^{\star}$. From top to bottom, we have: (1) $\lambda_{1}^{\star}<\dot{\lambda}_{1}$, (2) $\dot{\lambda}_{1} \leq \lambda_{1}^{\star}<\ddot{\lambda}_{1}$, (3) $\ddot{\lambda}_{1} \leq \lambda_{1}^{\star}<\bar{\lambda}_{1}$, and (4) $\bar{\lambda}_{1} \leq \lambda_{1}^{\star}$. The arrows indicate the direction of increasing $R_{\text {sum }}^{\mathrm{STRS}}$.

where

$$
\left(\lambda_{1}^{\star \star}, \lambda_{2}^{\star \star}\right)= \begin{cases}\left.\left(\lambda_{1}^{\star}, 1-\frac{\beta_{1}}{\beta_{2}}\left(1-\lambda_{1}^{\star}\right)\right)\right) & ,\left\{\begin{array}{l}
D>0 \\
\dot{\lambda}_{1} \leq \lambda_{1}^{\star}<\check{\lambda}_{1}
\end{array}\right. \\
\left(\check{\lambda}_{1}, 1-\frac{\beta_{1}}{\beta_{2}}\left(1-\check{\lambda}_{1}\right)\right) & ,\left\{\begin{array}{l}
D>0 \\
\max \left(0, \dot{\lambda}_{1}\right)<\check{\lambda}_{1} \leq \lambda_{1}^{\star}
\end{array}\right. \\
\left(0, \min \left(\tilde{\lambda}_{2}, 1-\frac{\beta_{1}}{\beta_{2}}\right)\right) & , \text { otherwise, }\end{cases}
$$

with $\lambda_{1}^{\star}=\max \left(0, \lambda^{\star}\right)\left(\lambda^{\star}\right.$ from (33)), $\tilde{\lambda}_{1}$ and $\tilde{\lambda}_{2}$ from (55), $\check{\lambda}_{1}=\min \left\{\bar{\lambda}_{1}, \ddot{\lambda}_{1}\right\}$, and $D, \dot{\lambda}_{1}, \ddot{\lambda}_{1}$ and $\bar{\lambda}_{1}$, as detailed in the next subsection. The optimum parameter settings are $\left(\iota ; \lambda_{1}, \lambda_{2}\right)=\left(1 ; \lambda_{1}^{\star \star}, \lambda_{2}^{\star \star}\right)$ if the maximum in (56) is equal to $r_{1 ; b}\left(\lambda_{1}^{\star \star}, \lambda_{2}^{\star \star}\right)$. Otherwise, the optimum parameter are $\left(\iota ; \lambda_{1}, \lambda_{2}\right)=\left(2 ; \min \left(\tilde{\lambda}_{1}, 1\right), 0\right)$ if $\gamma_{1,1} \geq \gamma_{2,1}$, and $\left(\iota ; \lambda_{1}, \lambda_{2}\right)=(2 ; 0,0)$, if $\gamma_{1,1} \leq \gamma_{2,1}$.

\section{F. Closed-form expressions for $D, \bar{\lambda}_{1}, \dot{\lambda}_{1}$ and $\ddot{\lambda}_{1}$}

In Fig. 6, the point at which the arc $a_{2}$ (with prescription $\left.\lambda_{2}=\mathcal{A}_{2}\left(\lambda_{1}\right)\right)$ intersects with the ray $\overrightarrow{F E}$ has $\lambda_{1}$-coordinate $\bar{\lambda}_{1}$, and the points at which the arc $a_{1}$ (with prescription $\lambda_{1}=\mathcal{A}_{1}\left(\lambda_{2}\right)$ ) intersects with the line $F E$ (with prescription $\lambda_{2}=1-\frac{\beta_{1}}{\beta_{2}}\left(1-\lambda_{1}\right)$ ) have $\lambda_{1}$-coordinates $\dot{\lambda}_{1}$ and $\ddot{\lambda}_{1}$, with $\dot{\lambda}_{1}<\ddot{\lambda}_{1}$. Using (24) and (46), it is easily found that $\dot{\lambda}_{1}=\dot{\lambda}\left(\gamma_{1,1}, \gamma_{1,2}, \gamma_{2,1}, \gamma_{2,2}\right), \ddot{\lambda}=\ddot{\lambda}\left(\gamma_{1,1}, \gamma_{1,2}, \gamma_{2,1}, \gamma_{2,2}\right)$ and $\bar{\lambda}_{1}=\bar{\lambda}\left(\gamma_{1,1}, \gamma_{1,2}, \gamma_{2,1}, \gamma_{2,2}\right)$, with (as $\beta_{2} \geq \beta_{1}>0$ in Fig. 6):

$$
\bar{\lambda}_{1}=\frac{-b-\sqrt{b^{2}-4 a c}}{2 a},
$$

$\dot{\lambda}_{1}=\frac{-B-\sqrt{D}}{2 A}, \ddot{\lambda}_{1}=\frac{-B+\sqrt{D}}{2 A}$, with $D=B^{2}-4 A C$

where

$$
a=-\gamma_{2,1}\left(\gamma_{1,2} \frac{\beta_{1}}{\beta_{2}}+\gamma_{1,1}\right)
$$




$$
\begin{gathered}
b=\gamma_{1,2} \frac{\beta_{1}}{\beta_{2}}\left(2 \gamma_{2,1}+\gamma_{2,2}\right)-\gamma_{2,1}-\gamma_{1,2} \gamma_{2,1}-\gamma_{1,1} \\
c=\frac{\left(\gamma_{2,1}+\gamma_{2,2}\right)\left(\gamma_{2,2}-\gamma_{1,2}\right)\left(1+\gamma_{1,1}+\gamma_{1,2}\right)}{\beta_{2}} \\
A=-\gamma_{1,2} \frac{\beta_{1}}{\beta_{2}}\left(\gamma_{2,2} \frac{\beta_{1}}{\beta_{2}}+\gamma_{2,1}\right) \\
\frac{B=\gamma_{1,1} \gamma_{2,1}+2\left(\frac{\beta_{1}}{\beta_{2}}\right)^{2} \gamma_{1,2} \gamma_{2,2}+}{\beta_{2}}\left(\gamma_{1,2} \gamma_{2,1}-2 \gamma_{1,2} \gamma_{2,2}-\gamma_{1,2}-\gamma_{2,2}\right) \\
C=\gamma_{1,1}-\gamma_{2,2}-\gamma_{1,2} \gamma_{2,2}+ \\
\frac{\beta_{1}}{\beta_{2}}\left(\gamma_{1,2}+\gamma_{2,2}+2 \gamma_{1,2} \gamma_{2,2}-\frac{\beta_{1}}{\beta_{2}} \gamma_{1,2} \gamma_{2,2}\right) \\
\text { REFERENCES }
\end{gathered}
$$

\section{REFERENCES}

[1] B. Clerckx, H. Joudeh, C. Hao, M. Dai, and B. Rassouli, "Rate splitting for MIMO wireless networks: A promising PHY-layer strategy for LTE evolution," IEEE Commun. Mag., vol. 54, no. 5, pp. 98-105, 2016.

[2] S. Chatzinotas, B. Ottersten, and R. De Gaudenzi, Cooperative and cognitive satellite systems. Academic Press, 2015.

[3] A. El Gamal and Y.-H. Kim, Network information theory. Cambridge University Press, 2011.

[4] W. Yu and T. Lan, "Transmitter optimization for the multi-antenna downlink with per-antenna power constraints," IEEE Trans. Signal Process., vol. 55, no. 6, pp. 2646-2660, 2007.

[5] H. Joudeh and B. Clerckx, "Sum-rate maximization for linearly precoded downlink multiuser MISO systems with partial CSIT: A rate-splitting approach," IEEE Trans. Commun., vol. 64, no. 11, pp. 4847-4861, Nov 2016.

[6] A. G. Davoodi, B. Yuan, and S. A. Jafar, "GDoF region of the MISO BC: Bridging the gap between finite precision and perfect CSIT," IEEE Trans. Inf. Theory, vol. 64, no. 11, pp. 7208-7217, 2018.

[7] S. Yang, M. Kobayashi, D. Gesbert, and X. Yi, "Degrees of freedom of time correlated MISO broadcast channel with delayed CSIT," IEEE Trans. Inf. Theory, vol. 59, no. 1, pp. 315-328, 2013.

[8] T. Han and K. Kobayashi, "A new achievable rate region for the interference channel," IEEE Trans. Inf. Theory, vol. 27, no. 1, pp. 49-60, Jan 1981.

[9] H. Joudeh, X. Yi, B. Clerckx, and G. Caire, "On the optimality of treating inter-cell interference as noise: Downlink cellular networks and uplink-downlink duality," IEEE Transactions on Information Theory, vol. 66, no. 11, pp. 6939-6961, 2020.

[10] H. Joudeh and G. Caire, "Cellular Networks With Finite Precision CSIT: GDoF Optimality of Multi-Cell TIN and Extremal Gains of Multi-Cell Cooperation," arXiv preprint arXiv:2008.08945, 2020.

[11] B. Clerckx and D. Gesbert, "Space-Time encoded MISO broadcast channel with outdated CSIT: An error rate and diversity performance analysis," IEEE Trans. Commun., vol. 63, no. 5, pp. 1661-1675, 2015.

[12] M. A. Maddah-Ali and D. Tse, "Completely stale transmitter channel state information is still very useful," IEEE Trans. Inf. Theory, vol. 58, no. 7, pp. 4418-4431, 2012.

[13] C. Hao, Y. Wu, and B. Clerckx, "Rate analysis of two-receiver MISO broadcast channel with finite rate feedback: A rate-splitting approach," IEEE Trans. Commun., vol. 63, no. 9, pp. 3232-3246, 2015.

[14] M. Payaró, X. Mestre, A. Pérez-Neira, and M. A. Lagunas, "Robust power allocation techniques for MIMO systems under modulus channel knowledge at the transmitter," in IEEE 6th Workshop on Signal Processing Advances in Wireless Communications, New York, NY, USA, June 2005, pp. 690-694.
[15] A. G. Davoodi and S. A. Jafar, "Transmitter cooperation under finite precision CSIT: A GDoF perspective," IEEE Trans. Inf. Theory, vol. 63, no. 9, pp. 6020-6030, 2017.

[16] N. Jindal and Z.-Q. Luo, "Capacity limits of multiple antenna multicast," in IEEE International Symposium on Information Theory (ISIT), vol. 6, Seattle, Washington, USA, 2006, pp. 1841-1845.

[17] T. Ramírez, C. Mosquera, M. Caus, A. Pastore, M. Navarro, and N. Noels, "Message-splitting for interference cancellation in multibeam satellite systems," in 9th Advanced Satellite Multimedia Systems Conference and the 15th Signal Processing for Space Communications Workshop (ASMS/SPSC), Berlin, Germany, 2018.

[18] A. Gholami Davoodi, "Aligned Image Sets: Instruments for Robust GDoF Bounds," Ph.D. dissertation, UC Irvine, 2018.

[19] J. Chen and P. Elia, "Toward the performance versus feedback tradeoff for the two-user MISO broadcast channel," IEEE Transactions on Information Theory, vol. 59, no. 12, pp. 8336-8356, 2013.

[20] H. Joudeh and B. Clerckx, "Robust Transmission in Downlink Multiuser MISO Systems: A Rate-Splitting Approach," IEEE Trans. Signal Process., vol. 64, no. 23, pp. 6227-6242, 2016.

[21] S. A. Jafar and A. J. Goldsmith, "Isotropic fading vector broadcast channels: The scalar upper bound and loss in degrees of freedom," IEEE Trans. Inf. Theory, vol. 51, no. 3, pp. 848-857, 2005.

[22] M. Vu, "MISO capacity with per-antenna power constraint," IEEE Trans. Commun., vol. 59, no. 5, pp. 1268-1274, 2011.

[23] R. H. Etkin, D. N. C. Tse, and H. Wang, "Gaussian interference channel capacity to within one bit," IEEE Trans. Inf. Theory, vol. 54, no. 12, pp. 5534-5562, Dec 2008.

[24] O. Mehanna, J. Marcos, and N. Jindal, "On achievable rates of the twouser symmetric gaussian interference channel," in 48th Annual Allerton Conference on Communication, Control, and Computing, Allerton House, Illinois, USA, 2010, pp. 1273-1279.

[25] D. K. Shin and W. Choi, "Optimal rate splitting in the two-user symmetric gaussian interference channel," in Seventh International Conference on Ubiquitous and Future Networks, Sapporo, Japan, 2015, pp. 662-666.

[26] A. Haghi and A. K. Khandani, "The maximum Han-Kobayashi sumrate for Gaussian interference channels," in 2016 IEEE International Symposium on Information Theory (ISIT), Barcelona, Spain, July 2016, pp. 2204-2208.

[27] B. Clerckx, Y. Mao, R. Schober, and H. V. Poor, "Rate-splitting unifying SDMA, OMA, NOMA, and multicasting in MISO broadcast channel: A simple two-user rate analysis," IEEE Wireless Commun. Lett., vol. 9, no. 3, pp. 349-353, 2020.

[28] H. Joudeh and B. Clerckx, "Rate-splitting for max-min fair multigroup multicast beamforming in overloaded systems," IEEE Trans. Wireless Commun., vol. 16, no. 11, pp. 7276-7289, 2017.

[29] Y. Mao, B. Clerckx, and V. O. Li, "Rate-splitting multiple access for downlink communication systems: bridging, generalizing, and outperforming SDMA and NOMA," EURASIP J Wirel Commun Netw, vol. 2018, no. 1, p. 133, 2018.

[30] A. G. Davoodi and S. Jafar, "Optimality of simple layered superposition coding in the 3 user MISO BC with finite precision CSIT," IEEE Trans. Inf. Theory, vol. 65, no. 11, pp. 7181-7207, 2019.

[31] Z. Li, C. Ye, Y. Cui, S. Yang, and S. Shamai, "Rate splitting for multiantenna downlink: Precoder design and practical implementation," IEEE Journal on Selected Areas in Communications, vol. 38, no. 8, pp. 1910 1924, 2020.

[32] M. Dai, B. Clerckx, D. Gesbert, and G. Caire, "A rate splitting strategy for massive MIMO with imperfect CSIT," IEEE Trans. Wireless Commun., vol. 15, no. 7, pp. 4611-4624, 2016.

[33] D. Taleb, Y. Liu, and M. Pesavento, "Full-rate general rank beamforming in single-group multicasting networks using non-orthogonal STBC," in 24th European Signal Processing Conference (EUSIPCO), Budapest, Hungary, 2016, pp. 2365-2369. 